\title{
Antioxidant Potential of Pediococcus Pentosaceus Strains from the Sow Milk Bacterial Collection in Weaned Piglets
}

\section{Leli Wang}

Hunan Normal University

Qihang Liu

Hunan Normal University

\section{Yuwei Chen}

Hunan Normal University

\section{Xinlei Zheng}

Hunan Normal University

\section{Chuni Wang}

Hunan Normal University

\section{Yining Qi}

Hunan Normal University

\section{Yachao Dong}

Hunan Normal University

\section{Yue Xiao}

Hunan normal university

\section{Cang Chen}

Hunan Normal University

\section{Taohong Chen}

Hunan Normal University

\section{Qiuyun Huang}

Hunan Normal University

Jialu Huang

Hunan Agricultural University

Zongzhao Zhai

Hunan Normal University

Cimin Long

Institute of Subtropical Agriculture Chinese Academy of Sciences

\section{Huansheng Yang}

Hunan Normal University

Jianzhong Li 
Hunan Normal University

\section{Lei Wang}

Hunan Normal University

\section{Gaihua Zhang}

Hunan Normal University

\section{Peng Liao}

Institute of Subtropical Agriculture Chinese Academy of Sciences

\section{Yongxin Liu}

Institute of Genetics and Developmental Biology CAS: Institute of Genetics and Developmental Biology Chinese Academy of Sciences

\section{Peng Huang}

Hunan Agricultural University

\section{Qiye Wang}

Hunan Normal University

\section{Huanhuan Chu}

Yantai Hi-tech Industrial Development Zone

Jia Yin ( $\square$ jiayin@hunnu.edu.cn )

animal nutrition and human health laboratory https://orcid.org/0000-0002-8867-3490

\section{Yulong Yin}

Institute of Subtropical Agriculture Chinese Academy of Sciences

\section{Research}

Keywords: culturomics, sow milk, probiotic, oxidative stress, Drosophila, piglets

Posted Date: November 1st, 2021

DOl: https://doi.org/10.21203/rs.3.rs-75522/v2

License: (c) (1) This work is licensed under a Creative Commons Attribution 4.0 International License. Read Full License 


\section{Antioxidant Potential of Pediococcus Pentosaceus Strains from} the Sow Milk Bacterial Collection in Weaned Piglets

Leli Wang ${ }^{1}$, Qihang Liu ${ }^{1}$, Yuwei Chen ${ }^{1}$, Xinlei Zheng ${ }^{1}$, Chuni Wang ${ }^{1}$, Yining Qi ${ }^{1}$,

Yachao Dong ${ }^{1}$, Yue Xiao ${ }^{1}$, Cang Chen ${ }^{1}$, Taohong Chen $^{1}$, Qiuyun Huang ${ }^{1}$, Jialu Huang ${ }^{5}$,

Zongzhao Zhai ${ }^{1}$, Cimin Long ${ }^{2}$, Huansheng Yang ${ }^{1}$, Jianzhong Li $^{1}$, Lei Wang ${ }^{1}$, Gaihua

Zhang ${ }^{3}$, Peng Liao ${ }^{2}$, Yongxin Liu ${ }^{4}$, Peng Huang ${ }^{5}$, Qiye Wang ${ }^{1}$, Huanhuan $\mathrm{Chu}^{6}$, Jia

Yin $^{1^{*}}$, Yulong Yin ${ }^{2 *}$

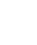

${ }^{1}$ Laboratory of Animal Nutrition and Human Health, Hunan Provincial Key Laboratory of Animal Intestinal Function and Regulation, Hunan International Joint Laboratory of Animal Intestinal Ecology and Health, College of Life Sciences, Hunan Normal University, Changsha, China.

${ }^{2}$ Institute of Subtropical Agriculture, Chinese Academy of Sciences, Changsha, China

${ }^{3}$ The National and Local Joint Engineering Laboratory of Animal Peptide Drug

Development, College of Life Sciences, Hunan Normal University, Changsha, China

${ }^{4}$ State Key Laboratory of Plant Genomics, Institute of Genetics and Developmental

Biology, Chinese Academy of Sciences, Beijing, China

${ }^{5}$ College of Animal Science and Technology, Hunan Agricultural University,

Changsha, Hunan, China.

${ }^{6}$ Shandong Yihe Feed Co.,Ltd. Yantai Hi-tech Industrial Development Zone, Yantai,

Shandong, China.

*Correspondence: jiayin@hunnu.edu.cn; yinyulong@isa.ac.cn 


\section{Abstract}

Background: In modern animal husbandry, breeders pay increasing attention to improving sow nutrition during pregnancy and lactation to favor the health of neonates. Sow milk is a main food for piglets during their first three weeks of life, which is not only a rich repository of essential nutrients and a broad range of bioactive compounds, but also an indispensable source of commensal bacteria. Maternal milk microorganisms are important sources of commensal bacteria for neonatal gut. Bacteria from maternal milk may serve as an additive to confer a health benefit on the composition of the indigenous microbiota of piglets.

Methods: The sow milk microbiota was collected using the culturomics methods of Continuous Culture and Interval Sampling, following by the identification of $16 \mathrm{~S}$ rDNA gene sequences. To screen potential probiotics, the functional evaluation was conducted to assess their antagonistic activity against pathogens in vitro and evaluate their resistance against oxidative stress in damaged Drosophila induced by paraquat. In piglet feeding trial, a total of 54 newborn suckling piglets were chosen from nine sows and randomly assigned to three treatments with different concentration of a candidate strain. Multiple approaches were carried out to verify its antioxidant function including western blotting, enzyme activity analysis, metabolomics and 16S rDNA sequencing.

Results: The 1240 isolates were screened out from the sow milk microbiota and grouped into 271 bacterial taxa based on a nonredundant set of 16S rDNA gene sequences. Among 80 Pediococcus isolates, a new Pediococcus pentosaceus strain 
(SMM914) showed best performance in inhibition ability against swine pathogens and in Drosophila model challenged by paraquat. Pretreatment of piglets with SMM914 induces the Nrf2-Keap1 antioxidant signaling pathway and greatly affected the pathways of amino acid metabolism and lipid metabolism in plasma. In colon, Lactobacillus was significantly increased in the high dose of SMM914 group compared with the control group.

Conclusion: SMM914 functions as a promising probiotic conferring antioxidant capacity by activating the Nrf2-Keap1 antioxidant signaling pathway in piglets. Our study provided useful resources for deeply understanding the relationships between the maternal microbiota and offspring.

Keywords : culturomics, sow milk, probiotic, oxidative stress, Drosophila, piglets 
60

61

62

\section{Introduction}

A unique microbiome is established in every individual in their first few years of life. In particular, the first year of life is a crucial stage of the development of the microbiome. Breastfeeding is the main factor affecting the development of the microbiome in this period [1]. Breastfed infants have a decreased risk of acute and chronic diseases, including gastroenteritis and sudden infant death syndrome, because breast milk provides essential nutrients and a broad range of bioactive compounds for developing neonates [2]. Commensal bacteria from breast milk act as pioneer bacteria during the critical stage of initial neonatal gut colonization [3]. Several studies at the strain level have specifically demonstrated that there are some bacteria shared between human breast milk and infant feces by isolating and identifying bacteria [4]. The vertical transfer concept that the breast milk microbiota can be transmitted from mothers to infants during breast feeding became accepted gradually [5]. The maternal microbiota contributes to the 'initial' intestinal microbiota establishment in infants and help to modulate both short- and long-term infant health outcomes [6-8].

Due to the high degree of similarity in anatomy, physiology and immunology between humans and pigs, piglets have been extensively used as an ideal model to study infants' gastrointestinal system and health [6]. Nowadays, pork is the most widely consumed meat in the world [9]. Early weaning is an abrupt event in piglets that often leads to severe oxidative stress and restricts the development of commercial swine husbandry. Breast milk is a main food for piglets during the first three weeks of their lives. 
Although abundant probiotics have been isolated from human milk and bovine milk [10-12], strategies for the preservation and isolation of the natural bacteria of sow milk are limited currently. While culture-independent methods have allowed an understanding of the composition and diversity of sow milk microbiota [9], culturedependent methods are still critical for the functional identification and utilization of the sow milk microbiota, which might be indirectly or directly beneficial for precise care of early-weaning piglets. However, no research has systematically investigated the sow milk microbiota using culture-dependent methods so far.

In the present work, a comprehensive collection of sow milk microbiota was first established by the culturomics of Continuous Culture and Interval Sampling. Among the 1,240 bacterial isolates, 80 strains of Pediococcus pentosaceus ( $P$. pentosaceus) were identified. $P$. pentosaceus, a gram-positive, catalase-negative, homofermentative bacteria strain that has long been used as biopreservatives and healthy commercial starters in fermented foods [13-15]. We depended on antagonistic experiments against pathogens in vitro and antioxidant tests in Drosophila model to select the candidate probiotic species Pediococcus pentosaceus ( $P$. pentosaceus). Interestingly, the antioxidant activity of $P$. pentosaceus SMM914 in piglets was verified by elevated antioxidant metabolites in plasma and the activation of the Nrf2-Keap1 pathway in livers. It is suggested that SMM914 could confer health benefits to piglets and represent a promising candidate for maintaining redox balance. These results of the sow milk 
bacterium and its antioxidant function in piglets deserve more attention for promoting healthy livestock breeding.

\section{Results}

\section{A comprehensive collection of lactic acid bacteria from sow milk}

Breast milk is one of common sources of probiotic strains [16]. We hypothesized that these lactic acid bacteria (LAB) from sow milk could facilitate adaptive, functional changes for the optimal weaning transition of piglets, which are vulnerable to various stressors in early life. However, the diversity and function of probiotics in porcine milk remain relatively understudied [17]. To establish a sow milk bacterial collection $(\mathrm{smBC})$, a large-scale cultivation and identification of the sow milk microbiota (SMM) were performed by sow milk collection, anerobic incubation and sequencing (Fig. 1, steps 1-3).

After the first three steps, we obtained 1240 isolates derived from the sow milk microbiota by the culturomics of Continuous Culture and Interval Sampling. These isolates were grouped into 271 bacterial taxa in the CD-HIT analysis based on a nonredundant set of sequences of the V1-V5 region of $16 \mathrm{~S}$ rDNA gene with a cutoff value of $99 \%$ identity for classification. The Silva version $13216 \mathrm{~S}$ rRNA database, NCBI nucleotide collection (nr/nt) database and DAIRYdb reference database were used to classify these prokaryotes into two categories: suspected new species and previously identified prokaryote species (including known species from dairy products).

A phylogenetic tree was built based on the calculated distances between pairs of 
sequences (Suppl. Data 1). The results showed that 151 taxa were assigned to

123 previously described species (black, Fig. S1), while the other 120 taxa could not be assigned to any known species (blue, Fig. S1), which were defined as suspected new species. The alignment against the DAIRYdb reference database revealed that 107 out of the 271 taxa were assigned to species found in dairy products (red dots, Fig. S1).

Specifically, twenty-three species were assigned to previously described species found in both the DAIRYdb reference database and Silva version 132 16S rRNA sequences database or the NCBI nucleotide collection (nr/nt) database, including A. lwoffii, Acinetobacter sp. , C. perfringens, Pelomonas, Enterococcus sp., E. durans, L. amylovorus, L. taiwanensis, L. garvieae, L. mesenteroides, Enterobacteriaceae bacterium DHL-32, Lactobacillales bacterium, Lactobacillaceae bacterium, Streptococcaceae bacterium, S. enterica and Salmonella sp. Nine species were suspected new species and also could not be assigned to any known species in the DAIRYdb reference database. In addition, five species were assigned to a previously described species in the Silva version 132 16S rRNA database or NCBI nucleotide collection (nr/nt) database, but not in the DAIRYdb database, including Acidovorax sp. SEPRH9, S. hyovaginalis, S. mitis, Streptococcus sp. S2 and S. thoraltensis (Fig. 2). A total of 922 out of 1240 isolates belong to Lactobacillales (Suppl. Data 2), and this group was dominated by Lactococcus lactis, which was roughly consistent with the microbiota composition of sow milk during lactation according to a recent sequencing report [17]. The genera Staphylococci and Streptococci represented 5.81\% and 4.03\% 
143 of the total bacterial isolates, respectively. These results supported a view that

144 commensal Staphylococci and Streptococci commonly occur in breast milk [18, 19],

145 which may originate from the maternal skin [20]. The collection of 1240 isolates

146 obtained through these efforts provides insight into the diversity of sow milk microbiota

147 and enables more studies on their function associated with mammalian health.

148 Screening of candidate probiotic $P$. pentosaceus strains

149 To explore health-promoting bacterial genera, which are not limited to traditional

150 probiotics of Lactobacillus and Bifidobacterium, we focused on Pediococcus.

151 Antagonistic activity against pathogens to control their spread is a prerequisite for a

152 potential probiotic [21]. Piglets commonly encounter pathogens on farms at increasing

153 frequencies [22], including Salmonella typhimurium (S. typhimurium) [23],

154 enterohemorrhagic Escherichia coli (EHEC) [24], enterotoxigenic Escherichia coli

155 (ETEC) [21], Klebsiella pneumoniae (K. pneumoniae) [25], Aeromonas punctate (A.

156 punctate) [26], Staphylococcus aureus (S. aureus) [27], Listeria monocytogenes (L.

157 monocytogenes) [2] and Clostridium perfringens (C. perfringens) [28]. The fluctuating

158 size of the inhibition zone against pathogens indicates the inhibitory activities of

159 different $P$. pentosaceus isolates against both gram-negative and gram-positive

160 pathogenic bacteria (Fig. 3a), revealing strain-specific antimicrobial activity against

161 different bacteria [29]. The morphology of the top ten strains was observed (Fig. S2),

162 and they were then used in subsequent tests. 
For these $P$. pentosaceus strains with strong antimicrobial activity, we next used a simple animal model to rapidly screen bacteria that show potent antioxidant activity in vivo (Fig. 3b). Considering the similarities of the intestinal development with mammals and the cost of the mouse model $[30,31]$, Drosophila could be an appropriate model to evaluate the ability of more bacteria in protection of hosts from reactive oxygen species (ROS), whose accumulation cause damage to the health of both Drosophila and mammals $[30,32]$. In this study, resistance to paraquat was used as a measure of free radical scavenging activity in the Drosophila system [32]. After paraquat treatment for 45 hours, the flies colonized with $P$. pentosaceus SMM914 showed a significantly elevated survival rate in response to paraquat challenge ( $p<0.05$, log-rank test) (Fig. $3 \mathbf{c})$.

SMM914 showed a strong ability to inhibit the proliferation of $S$. aureus, while almost one-third of $P$. pentosaceus strains exhibited no antimicrobial ability toward this pathogen. Through the sodium hydroxide neutralization reaction, the antibacterial effect of SMM914 against S. aureus and ETEC was abolished, which proved its bacteriostatic effect was mainly due to the presence of organic acids (Fig. S3). The time curves for growth and $\mathrm{pH}$ of SMM914 were measured. The results revealed that the strain entered the stationary phase after $12 \mathrm{~h}$ of fermentation, while the $\mathrm{pH}$ was stabilized at approximately 3.9 after 36 h of fermentation (Fig. S4 a, b). A whole genome of SMM914 was sequenced, which generated 28 contigs and a total size of 
sequences, 62 RNA (including 54 tRNAs, 8 rRNAs), and 2 CRISPRS were identified in NCBI prokaryotic genome annotation pipeline. By analyzing the genome (Fig. S5), we obtained some clues about its probiotic properties. Genes related to organic acid, thioredoxin, and exopolysaccharide, which have potential of disease-resistance [33] or oxidative stress resistance activities [34, 35], were annotated in the genome (Suppl.

\section{Data 3).}

\section{Growth performance and serum biochemical parameters in the pig-feeding trial}

Because SMM914 showed bacteriostatic activity and the robust resistance to paraquatinduced stress in Drosophila, it was selected to feed the piglets in low-dose (LD) or high-dose (HD) groups prior to early weaning (Fig. 4a). Regarding visceral indices, a higher heart coefficient, which is the relative weight of the heart [36], was observed in piglets treated with SMM914 ( $p<0.05, n=7$ ) (Fig. S6). The heart coefficient has been reported to be negatively associated with oxidative stress via changes in angiotensin IIaldosterone-brain natriuretic peptide [36]. We speculated that the increased heart coefficient observed in this study could be an indicator of alleviated stress.

When constantly challenged by various endogenous or exogenous free radicals, the liver in vertebrates is susceptible to damages [23, 37]. Weaning is frequently associated with liver injury [38]. Results of blood biochemical parameters revealed that total protein (TP) and albumin (ALB) levels decreased in low dose of SMM914 (Fig. S7). Moreover, treatment with SMM914 no matter in low concentration or high 
(LDH) levels (Fig. 4b). The elevation of ALT and LDH activities in serum can be used

as biomarkers of hepatic disorders under oxidative stress [39, 40]. Our findings demonstrated that SMM914 administration led to an amelioration on liver injury during weaning.

Pretreatment of piglets with SMM914 induces the Nrf2-Keap1 antioxidant

\section{signaling pathway}

To discover the molecular mechanisms that might underlie the antioxidative effect, we investigated the alteration of the nuclear factor (erythoid-derived)-like 2 ( $\mathrm{Nrf} 2$ ) signaling pathway, a conserved signaling pathway across metazoans [41], in the liver of piglets by western blotting analysis and enzyme activity assays. Kelch-like ECHassociated protein 1 (Keap1) is a specific repressor of Nrf2 via tight binding. Antioxidant metabolites can contribute to the dissociation of Keap1 and Nrf2 complex, promoting Nrf2 movement into the nucleus. Nrf2 transfers from the cytosol to the nucleus, resulting in the coordinated transcriptional upregulation of a battery of the intranuclear protein expression level of Nrf2 but also led to elevated protein levels of NADPH quinineoxidoreductase-1 (NQO-1), catalase (CAT), hemeoxygenase-1 antioxidant enzymes and detoxifying proteins [42]. As expected, in the western blotting analysis of this study, the protein level of Keap1 was remarkably suppressed in piglets receiving SMM914 $(p<0.05)$. We found that SMM914 not only markedly increased of NADPH quinineoxidoreductase-1 (NQO-1), catalase (CAT), hemeoxygenase-1 (HO-1) and superoxide dismutase (SOD) in a concentration-dependent manner (Fig. 
4c-f \& Fig. S8). CAT and SOD are widely recognized as important endogenous antioxidant enzymes that scavenge hydroxyl and superoxide anion radicals [43, 44]. In the enzyme activity assays, the HD group simultaneously increased glutathione peroxidase (GSH-Px) activity, CAT activity and SOD activity $(p<0.05)$ in livers (Fig. 4g). Additionally, the HD group showed a significant decrease in malondialdehyde (MDA), a product of lipid peroxidation, in livers compared with the control group. The western blotting data combined with enzyme activity tests suggested that SMM914 functions as a promising probiotic conferring antioxidant capacity through activation of the Nrf2-Keap1 antioxidant signaling pathway in piglets.

\section{The altered pathways of amino acid metabolism and lipid metabolism in plasma}

To provide a better understanding of the antioxidant effect of SMM914, we further examined the metabolic profiles of blood plasma from the three groups $(n=7)$. PLSDA plots showed separated clusters with an optimal goodness of fit $\left(\mathrm{R}^{2}=0.996, \mathrm{Q} 2=\right.$ 0.681 (Fig. 5a); $\mathrm{R}^{2}=0.994, \mathrm{Q} 2=0.479$ (Fig. 5b)), indicating that the models were suitable and reliable for prediction. The KEGG enrichment of differential metabolites revealed that the pathways of lipid metabolism and amino acid metabolism were the main perturbed metabolic pathways between the HD group and the control group. The possible protective effect of SMM914 on the weaned piglets is depicted in Fig. 5c.

243 In the lipid metabolism, decreased levels of cortisol and corticosterone (Fig. 5d) were 244 observed in plasma, which are classic stress hormones and related to steroid hormone 
$\overline{\text { Under psychological and emotional stress conditions, corticosterone and cortisol }}$

247 induce the oxidative load in the brain, with a significant increase in pro-oxidant markers

248 in constantly changing environments [46]. After maternal separation, separation

249 anxiety in human infants is an inevitable phenomenon at weaning that may raise cortisol

250 levels and even alter the gut microbiota composition through the gut-brain axis [47,

251 48]. Meanwhile, the intensities of cholic acid, taurochenodeoxycholate and

252 glycochenodeoxycholate (Fig. 5e) were also decreased in the LD group to 0.43 -fold ( $p$

$253<0.05), 0.58$-fold $(p=0.08)$ and 0.47 -fold $(p<0.05)$, respectively, compared to the

254 levels in the control.

255 Conversely, in amino acid metabolism, several critical antioxidant metabolites

256 (cysteine-S-sulfate, DL-methionine sulfoxide, L-methionine) closely related to cysteine

257 and methionine metabolism were significantly increased to 1.41-2.03-fold in the HD

258 group compared with the control group $(p<0.05)$ (Fig. 5f). D-proline, L-proline and

259 L-glutamate, which are involved in arginine and proline metabolism, were increased in

260 the LD group compared with the control group to 1.43-fold ( $p<0.05), 1.30$-fold $(p<$

$2610.05)$ and 1.34-fold $(p=0.056)$, respectively (Fig. 5g). In the glycine, serine and

262 threonine pathway, choline was also significantly increased to 1.18 -fold in the LD

263 group $(p<0.05)$ compared with the control group (Fig. 5h). These three amino acid

264 pathways are closely overlapped through several metabolites (Fig. 5c).

265 The reshaped colon microbiota in piglets by SMM914

266 The changeover from milk to solid feed strongly influences the development of the gut

267 microbiota [49]. Disorders in the composition of the microbiota can induce oxidative 
stress and chronic metabolic diseases through a liver-gut axis $[50,51]$. In this study,

colonic microbiota was further investigated by using 16S rDNA gene amplicon sequencing. No differences were observed among the control and treated groups in terms of $\alpha$-diversity (Fig. S9 a). All samples from weaned piglets approached the saturation plateau based on Shannon-Wiener rarefaction curves (Fig. S9 b), suggesting that the sampling was sufficient for nearly all bacterial species. The shared and specific genera are shown in a Venn diagram (Fig. 6a). The bacterial community of the three groups shared 89 genera. There were 20 unique genera in the HD group, including Akkermansia. Eleven genera, including Dorea and Lachnospiraceae_AC2044_group, were detected in the LD and HD groups but not in the control group. To intuitively measure the extent of the similarity of the overall microbiota, the results of PCA based on distance revealed a separate clustering of samples between the HD group and the control group ( $p=0.001$, ANOSIM, comparison between the HD and control samples), but the colonic specimens of the LD group were not separated from the control group ( $p=0.274$, ANOSIM, comparison between the LD and control samples). These results indicated that the high dose of SMM914 reshaped the microbiota structure of the colon (Fig. 6b). The HD group showed distinct bacterial communities compared to the control group both at family and genus level (Fig. 6c and Fig. S11). At the family level, the relative 287 abundance of the family Lactobacillaceae $(p<0.05)$ and the genus Lactobacillus $(p<$ 288 0.05) were both increased in HD group compared with the control group (Fig. 7a). For 
the family Christensenellaceae and Ruminococcaceae, which are beneficial bacteria reported before $[52,53]$, the means of relative abundance in the LD group and HD group were increased by $89.57 \%, 110.27 \%$ and $804.9 \%, 21.59 \%$, respectively,

292 compared with the control group (Fig. S10). The genus level, the relative abundances of Bacteroidaceae in the HD group were decreased by $86.7 \%$ (Fig. S11). Specifically, the genus Bacteroides was observed to decrease in the HD group compared with the control group $(p<0.05)$.

\section{Discussion}

In recent years, breeders have attached great importance to improving sow nutrition during pregnancy and lactation from the source, rather than merely purchasing economic benefits. Here, we put forth the concept of Sow and Piglet Integration (SPI), which is an integral nutritional regulation scheme based on the physiological stages of sows and piglets on pig farms and the interrelationship of the microbiota between the potential pathogens, with a direct impact on breeders' profitability [55]. To face 
in American have extended the lactation period to 25 days. Another promising alternative to antibiotics in animal feed is using probiotics to improve the absorption of the nutrients [56]. During the initial development of mammalian neonates, breast milk was a nutritious food and a natural reservoir of probiotics $[57,58]$ that adapted to satisfy neonatal needs in evolution [59]. In the present work, we focused on sow milk microbiota by using the culturomics of Continuous Culture and Interval Sampling. This identification work is an important step that could reveal the LAB repertoire of sow milk to analyze the relationships between the maternal microbiota and that of the offspring and to deeply understand the concept of SPI.

Nowadays, metagenomic approaches have generated countless sequences that have not reported to double the species number of microorganisms isolated from the human gut [60]. Although the culturomics approach is time and labor consuming, it is a more challenging task to evaluate the functional properties of such enormous isolates. To screen probiotics from sow milk quickly, we first evaluated their antagonistic activity against pathogens in vitro. Next, we selected several strains and further explored their antioxidant capacities in vivo. A rapid Drosophila screening model combined with a subsequent piglet experiment was used to screen out suitable probiotics from a collection of sow milk microbiota. In piglets treated with P. pentosaceus SMM914, we observed decreased ALT and TP levels. The shift observed in our study was in accordance with another report that the administration of $P$. pentosaceus LI05 significantly prevented increases in TP and ALT in the context of acute liver failure [61]. Besides, activation of the Nrf2-Keap1 signaling pathway was observed by western blotting analysis and enzyme activity assays in the liver of piglets. Nrf2 and its 
$\overline{\text { endogenous inhibitor, Keap1, are conserved defense mechanism for regulating }}$ antioxidative activities [62].

337 In untargeted metabolism of plasma, several metabolic pathways were perturbed, and they can be mainly classified into lipid metabolism and amino acid metabolism. In the cysteine and methionine metabolism, L-methionine is a limiting amino acid in early lactation [63] associated with various key physiologic events. The increased availability of L-methionine in early lactation was reported to have positive effects on plasma lipid metabolism and overall antioxidant status [64]. Methionine sulphoxide is also biologically available as a methionine source. High methionine bioavailability is likely Adenosyl-L-methionine is then used to generate S-Methyl-5'-thioadenosine and 1Aminocycloproane-1-carboxylic acid. Through the transsulfuration and transmethylation pathway, L-methionine is converted into L-cysteine which can serve as a substrate for glutathione synthesis. Glutathione is an endogenous sulfur-containing antioxidant $[65,66]$. Another substrate for glutathione synthesis is glutamate. In mammals, L-glutamate is an abundant amino acid in milk that is required for the synthesis of glutathione and alleviates oxidative stress by increasing antioxidant enzyme activities [45, 67]. Glutathione is decomposed into L-gamma-glutamyl amino acid, and L-gamma-glutamyl amino acid is further converted to pyroglutamic acid. A high level of pyroglutamic acid reflected glutathione deficiency and could be an indicator of the oxidative state [68]. However, in our study, the concentration of pyroglutamic acid was significantly downregulated. Collectively, the altered intensities of these metabolites might be conducive to the accumulation of glutathione. The cysteine and methionine metabolism is overlapped with choline metabolism tightly 
$\overline{\text { because choline can serve as the substrate for L-methionine synthesis. Choline is an }}$ essential vitamin for humans and other mammals to regulate amino acid metabolism [69], particularly when L-methionine levels are not sufficient around parturition [70]. It has been established that choline deficiency induces the generation of ROS [71] and oxidative damage in rats [72], ruminants [73] and fishes [74]. Moreover, dietary supplementation with choline enhances the antioxidative capacity in pigs with intrauterine growth restriction [75]. New evidence has shown that choline deficiencyinduced oxidative damage was associated with changes in the transcription of antioxidant enzymes and Nrf2 signaling in the liver [74].

In the primary bile acid biosynthesis and the bile secretion pathways, there are variable for the induction of oxidative stress [76, 77]. Deoxycholic acid can combine with taurine or glycine to form taurochenodeoxycholate or glycochenodeoxycholate, respectively that stimulate the phosphorylation of NADPH oxidase and the formation and Ruminococcaceae UCG-005 gerera, while those of piglets in control group exhibit higher relative abundances of genera from Bacteroidaceae and Prevotellaceae families. 
The depletion of the Lactobacillus in the gut environment has been associated to

383 oxidative damage, and members of this genus are commonly used as probiotics in animal husbandry by upregulating the expression of glutathione reductase and glutathione S-transferase during the suckling period [82, 83]. Moreover, methionine was reported to attenuate oxidative stress in rats through high abundances of Lactobacillus and Lachnospiraceae [84]. The Ruminococcaceae family is always negatively related to disease severity [53]. The Christensenellaceae_R_7_group plays a positive role in intestinal immunomodulation [85]. On the contrary, Bacteroidaceae and Prevotellaceae have been proved to be associated with several diseases. For example, chitosan oligosaccharides increase the antioxidant capacity by inhibiting the abundance of Bacteroides and Prevotella [83]. The dysbiotic microbiota of inflammatory bowel disease (IBD) is mostly characterized by an increase in Prevotellaceae and a reduction in Ruminococcaceae [86]. Improving the cellular antioxidant potential is a promising approach for prevention of IBD. In this work, it appeared that SMM914 administration in piglets positively regulate intestinal microbiota during the transition of early-weaned stress.

\section{Conclusion}

400 Here, we focused on bacteria profile from sow milk by using the culturomics of 401 Continuous Culture and Interval Sampling and established a set of methods for rapid screening the beneficial microorganisms due to the importance of swine for biomedical 
research and agriculture. It was indicated that SMM914 could activate the Nrf2-Keap1 antioxidant signaling pathway in piglets and relieve oxidative stress by the elevated level of amino acid metabolites in plasma and the increased beneficial microbiota in colon, although we cannot provide comprehensive mechanistic linkages that might participate in the process. The study has shed light on the effects of sow milk microbiota for deeply understanding the relationships between the sow and offspring. However, researchers are needed to further strengthen the research on safety and stability assessments of SMM914 to pave the way for the application of maternal probiotics in animal husbandry.

\section{Methods}

414 Culture media and bacterial strain isolation.

415 A total of nine healthy second-parity sows with similar breeding dates raised on a pig 416 breeding farm (Changsha, China) were employed in this study. The sows received no 417 antibiotics within the 4 weeks prior to breast milk sampling. The areolar skin around 418 the teats was successively swabbed with alcohol tampons and warm saline-lubricated 419 sterile swabs. Using sterile tubes, fresh ordinary milk was collected from six sows during lactation (Fig. S1, step 1).

421 Considering that bacterial populations can survive through cell death and the recycling 422 of dead cells [87], the continuous culture and intermittent sampling was performed for 42330 days in an anaerobic incubator $\left(\mathrm{N}_{2}=90 \%, \mathrm{CO}_{2}=5 \%\right.$, and $\left.\mathrm{H}_{2}=5 \%\right)$ at $37^{\circ} \mathrm{C}($ Fig. 
S1, step 2). Using sow milk as an inoculum, MRS (Oxoid, Code\# CM0359, UK) [88], M17 (Oxoid, Code\# CM0817, UK) [88], TPY (Hopebio, Code\# HB0397, China) [89] and GYP (Hopebio, Code\# HB8539, China) [90] media were utilized to cover as much LAB diversity as possible. The bacterial cells were harvested every other day by centrifugation at $4000 \mathrm{~g}$ for $10 \mathrm{~min}$, and the cell pellets were resuspended in sterile normal saline. Then, the cells were spread on agar plates and anaerobically cultured in a DG250 Anaerobic workstation (DWS, UK) at $37^{\circ} \mathrm{C}$ for $24 \mathrm{~h}-72 \mathrm{~h}$. The identification of the isolates was first carried out by using morphological method. Colonies were streaked on agar plates, and all isolates were stocked in $25 \%(\mathrm{v} / \mathrm{v})$ glycerol broth at $80^{\circ} \mathrm{C}$ at the College of Life Science, Hunan Normal University, China (Fig. S1, step 4). SMM914 was deposited at the China General Microbiological Culture Collection Center (CGMCC20160).

\section{Characterization and classification of the isolated bacteria}

After isolation and purification, DNA was extracted from pure cultures. The V1-V5 region of 16S rDNA genes was amplified using Takara PrimerSTAR Max DNA Polymerase with a pair of LAB-specific primers, $15 \mathrm{f}$ (5'GCTCAGGAYGAACGCYGG -3') and 687r (5'- CACCGCTACACATGRADTTC-3') for the identification of the isolates [91]. The PCR-amplified products were identified by Sanger sequencing (Sangon Biotech Ltd., China). The sequencing error-prone areas (50 bp) at both ends were removed. The partial 16S rDNA sequences were searched 
against the NCBI nucleotide collection (nr/nt) database using BLASTN. The best match for each sequence was selected, and the taxonomy was assigned (Fig. S1, step 3).

The nonredundant set of 16S rDNA datasets was clustered by using CD-HIT with 447 sequence identity of 0.99 [92]. The phylogenetic relationship between isolates was 448 determined by aligning the nonredundant set of $16 \mathrm{~S}$ rDNA gene sequences to construct 449 a maximum-likelihood tree by using FastTree.

450 All 16S rDNA sequences alignment were aligned against the Silva version 132 16S 451 rRNA database, the NCBI nucleotide collection (nr/nt) database and the DAIRYdb reference database using BLASTN with a threshold of 1e-05 e-value, 99\% coverage and $99 \%$ cutoff. A suspected new species was defined below these values against the

Silva version 132 16S rRNA database and NCBI nucleotide collection (nr/nt) database [92]. The sequencing read data have been deposited in the National Center for Biotechnology Information Sequence Read Archive (Suppl. data S2).

\section{Antibacterial assay}

458 The following indicator strains were used: S. typhimurium ATCC 14028, EHEC ATCC 43894, ETEC O149: K88, K. pneumoniae ATCC 13883, A. punctata subsp. Caviae ATCC 15468, S. aureus ATCC 25923, L. monocytogenes ATCC 19115 and C. perfringens ATCC 13124.

462 Agar well diffusion assays [93] were utilized to evaluate antimicrobial activity against 463 these indicator pathogenic strains in vitro. Briefly, pathogens were grown in Luria464 Bertani (LB) broth at $37^{\circ} \mathrm{C}$ for $8 \mathrm{~h}$ and then diluted at a volume ratio of $20 \mu \mathrm{L}$ to $4 \mathrm{~mL}$ 
LB and mixed well. Fifty microliters of the diluted liquid were spread evenly on each

466

467

468

469

470

471

472

473

soft LB agar plate containing $0.8 \%$ agar. The residual liquid was evaporated on a ventilated clean bench. Next, holes were punched in each agar plate via sterile iron pipettes, with a depth of $6 \mathrm{~mm}$ and a diameter of $5 \mathrm{~mm}$. P. pentosaceus strains were grown in MRS broth at $37^{\circ} \mathrm{C}$ for $18 \mathrm{~h}$. Then, supernatants of fermentation broth were precisely added to the holes with a $30 \mu \mathrm{L}$ volume per well. The central well of each plate was filled with $30 \mu \mathrm{L}$ MRS broth as the negative control. After $48 \mathrm{~h}$ of incubation at $37^{\circ} \mathrm{C}$, antibacterial activity was observed as a halo of inhibition in the bacterial lawn formed around the sample, and the diameter of the zones of inhibition was measured. The evaluation of each sample was repeated in triplicate.

\section{Paraquat resistance assays in Drosophila}

The Drosophila experiments were conducted under a $12 \mathrm{~h}$ light: $12 \mathrm{~h}$ dark cycle at $25^{\circ} \mathrm{C}$ on cornmeal-molasses medium. Six-day-old mated female Drosophila $w^{1118}$ were collected under $\mathrm{CO}_{2}$ anesthesia and starved for $2 \mathrm{~h}$. Each group consisted of 3 vials, and each vial contained 20 female flies. In the bacterial association assays, the colonyforming units (CFUs) of $P$. pentosaceus were enumerated using MRS agar plates following standard microbiological procedures. Groups of adult female flies were colonized with pure cultures $\left(1 \times 10^{10} \mathrm{CFUs}\right)$ of the $P$. pentosaceus strains for 3 days, including SMM847, SMM853, SMM862, SMM867, SMM881, SMM906, SMM907, SMM908, SMM914 and SMM918 isolates. Distilled water without P. pentosaceus was used as a negative control. Then, these flies were transferred to vials containing 2 pieces 
of Whatman paper soaked with $200 \mu \mathrm{L} 5 \%(\mathrm{w} / \mathrm{v})$ sucrose containing $12 \mathrm{mmol} / \mathrm{mL}$

487 paraquat (methyl viologen dichloride, Cat\# 856177, Sigma-Aldrich, USA). Each group was supplied with fresh paraquat vials every day. The $12 \mathrm{mmol} / \mathrm{mL}$ concentration was chosen because it was empirically shown that this concentration was lethal to more than $80 \%$ of female $w^{1118}$ flies within 2 days.

\section{Piglet feeding trial and tissue sampling}

The Animal Care and Use Committee of the Institute of Subtropical Agriculture, Chinese Academy of Sciences, reviewed and approved the experimental procedures involving piglets. As described in Jun Hu et al [91], a total of 54 newborn suckling piglets (Landrace $\times$ Yorkshire) were chosen from nine second-parity sows and randomly assigned to three treatments $(n=18)$ among each litter, including a control group (physiological saline, $2.0 \mathrm{~mL}$ each time, Control), a low-dose SMM914 solution group $\left(10^{8} \mathrm{CFU} / \mathrm{ml}, 2.0 \mathrm{~mL}\right.$ each time, $\mathrm{LD}$ group $)$ and a high-dose SMM914 solution group $\left(10^{9} \mathrm{CFU} / \mathrm{ml}, 2.0 \mathrm{~mL}\right.$ each time, HD group). The solution of saline or bacterial cells was infused into each piglet's mouth by a syringe without a needle. All suckling piglets were subjected to oral gavage every other day from the age of 10 to 18 days and were weaned at 21 days.

\section{Sample collection}

Piglets per group from each of the 7 litters were selected to be euthanized for sampling at 28 days. Ten milliliters of blood were collected into heparin sodium anticoagulant tubes via direct cardiac puncture immediately after death and subjected to untargeted 
metabolism analyses. Another $10 \mathrm{~mL}$ of blood was collected in vacuum tubes and centrifuged at $3000 \mathrm{rpm}$ at $4^{\circ} \mathrm{C}$ for $10 \mathrm{~min}$. The serum samples were kept at $-80^{\circ} \mathrm{C}$ until analysis. After the opening of the abdomen, tissues including livers, spleens, kidneys, and hearts were weighed, dissected, and then snap-frozen in liquid nitrogen. Colon contents were stored at $-80^{\circ} \mathrm{C}$ until the extraction of bacterial DNA.

Serum concentrations of parameters reflecting lipid (cholesterol, triglyceride, highdensity lipoprotein cholesterol, low-density lipoprotein cholesterol, lipase), protein

514 (total protein, TP; albumin, ALB), carbohydrate (glucose, alpha-amylase, lactic dehydrogenase) metabolism, liver functionality including total bilirubin (TBIL), direct bilirubin (DBIL), indirect bilirubin (IBIL), the activity of aspartate transaminase (AST), alanine aminotransferase (ALT), gamma-glutamyl transpeptidase (GGT), serglobulin

518 (GLO), alkaline phosphatase (ALP), lactic dehydrogenase (LDH) and cholinesterase 519 (CHE), as well as kidney functionality including creatinine (CREA), blood urea nitrogen (BUN) and uric acid (UA) were determined using commercial kits according

521 to the manufacturer's instructions (Jiancheng Bioengineering Institute, Nanjing, China) 522 and were identified with a TBA-120FR Automatic Chemistry Biochemistry Hiiometer 523 (Hitachi Co., Tokyo, Japan).

\section{Western blotting analysis}

525 Western blotting was performed as previously described with some modification [82].

526 Liver samples $(n=6)$ were powdered under liquid nitrogen and lysed in 527 radioimmunoprecipitation assay buffer with the protease inhibitor 
phenylmethanesulfonyl fluoride (PMSF, Beyotime Biotechnology). The supernatant was obtained by centrifugation at $12,000 \times \mathrm{g}$ for $10 \mathrm{~min}$ at $4^{\circ} \mathrm{C}$. The denatured proteins were separated by $10 \%$ sodium dodecyl sulfate-polyacrylamide gel electrophoresis and then transferred to polyvinylidene fluoride membranes at $200 \mathrm{~mA}$ for $1 \mathrm{~h}$. The membranes were blocked with 5\% nonfat milk in Tris-buffered saline mixed with $0.5 \%$ Tween-20 (TBST) at room temperature for $2 \mathrm{~h}$ and then incubated with antibodies against Kelch-like ECH-associated protein 1 (Keap1) (SC-19917, Proteintech, USA), nuclear factor-E2-related factor 2 (Nrf2) (SC-98974, Proteintech, USA), NADPH quinineoxidoreductase-1 (NQO-1) (11451-1-AP, Proteintech, USA), hemeoxygenase1 (HO-1) (27282-1-AP, Proteintech, USA), catalase (CAT) (66765-1-Ig, Proteintech, USA), Cu/Zn-superoxide dismutase (SOD1) (10269-1-AP, Proteintech, USA), PCNA (60097-1-Ig, Proteintech, USA) or $\beta$-actin (SC-47778, Proteintech, USA), which were diluted with 5\% nonfat milk in TBST. The membranes were washed 3 times in TBST and then incubated with a secondary antibody. Finally, the membranes were washed with TBST solution and visualized with a chemiluminescence instrument.

\section{Enzyme activity analysis}

544 Liver tissue samples were homogenized in saline, followed by centrifugation $(2500 \times \mathrm{g}$,

$\left.5454^{\circ} \mathrm{C}, 10 \mathrm{~min}\right)$ to obtain supernatants $(n=7)$. Malondialdehyde (MDA), glutathione

546 peroxidase (GSH-Px) and superoxide dismutase (SOD) in livers were determined with 547 commercially available colorimetric diagnostic kits (Nanjing Jiancheng Bioengineering 
Institute, China) following the manufacturer's instructions. The procedures were carried out in duplicate with three parallel samples.

\section{Untargeted metabolomics}

551

552

The plasma samples $(100 \mu \mathrm{L})$ were thawed at $4{ }^{\circ} \mathrm{C}$ and homogenized in $400 \mu \mathrm{L}$ of precooled methanol/acetonitrile $(1: 1, \mathrm{v} / \mathrm{v})$ for $60 \mathrm{~s}$. The untargeted metabolic profiling analysis was conducted by using an ultra-performance liquid chromatography (UPLC) system (1290 Infinity LC, Agilent Technologies, Santa Clara, California, USA) coupled to a quadrupole time-of-flight (TOF) mass spectrometer (Triple TOF 5600, AB SCIEX) with electrospray ionization in positive and negative ionization modes. For chromatographic separation, $2 \mu \mathrm{L}$ of the extracted sample was injected by an autosampler system at $4^{\circ} \mathrm{C}$ at a delivery flow rate of $300 \mu \mathrm{L} / \mathrm{min}$ into a liquid chromatography column with a column temperature of $25^{\circ} \mathrm{C}$. The mobile phase consisted of $\mathrm{A}$ (water $+25 \mathrm{mM}$ ammonium acetate $+25 \mathrm{mM}$ ammonia hydroxide) and B (acetonitrile). The gradient was 95\% B and 5\% A for 1 min, with a linear reduction to $65 \% \mathrm{~B}$ and $35 \% \mathrm{~A}$ over $13 \mathrm{~min}$, a reduction to $40 \% \mathrm{~B}$ and $60 \% \mathrm{~A}$ over $2 \mathrm{~min}$, maintenance for $2 \mathrm{~min}$ and an increase to $95 \% \mathrm{~B}$ and $5 \% \mathrm{~A}$ over $0.1 \mathrm{~min}$, with a $5 \mathrm{~min}$ re-equilibration period. Before injection, quality control samples were used to monitor the stability and repeatability of the data produced by the instrument. The screening criteria for differential metabolites were based on a variable importance projection score $>1$ and $p<0.05$ (Student's t-test). The metabolites were analyzed by comparing the molecular ions with compounds in the available biochemical databases, and the 
pathway analysis of the identified compounds was conducted using the KEGG website

570

571

572

(http://www. genome. jp/kegg).

\section{S rDNA gene amplicon sequencing}

Intestinal digesta samples were collected after sacrifice. Bacterial DNA was extracted using a QIAamp DNA Stool Kit (Qiagen, Gaithersburg, MD, USA) according to the provided protocols $(n=7)$. The V3-V4 region of the bacterial 16S rDNA gene was amplified by PCR in triplicate in a $20 \mu \mathrm{L}$ mixture $\left(2 \mathrm{~min}\right.$ at $95^{\circ} \mathrm{C}$, followed by 25 cycles of $30 \mathrm{~s}$ at $95^{\circ} \mathrm{C}, 30 \mathrm{~s}$ at $55^{\circ} \mathrm{C}, 30 \mathrm{~s}$ at $72^{\circ} \mathrm{C}$ and $5 \mathrm{~min}$ at $\left.72^{\circ} \mathrm{C}\right)$. The primers $314-\mathrm{F}\left(5^{\prime}-\right.$ CCTAYGGGRBGCASCAG-'3) and 806-R (5'-GGACTACNNGGGTATCTAAT-'3) were used to target the hypervariable region. Using the AxyPrep DNA Gel Extraction Kit (Axygen, Union City, CA, USA), the PCR products were purified and then quantified by QuantiFluor ${ }^{\mathrm{TM}}$-ST (Promega, Madison, WI, USA). Library quality was assessed, and the amplicon library was sequenced on the Illumina MiSeq platform. The sequences were merged with FLASH ( v1.2.7) [94] and quality filtered with fastp (0.19.6) [95]. Then the high-quality sequences were de-noised using DADA2 [96] plugin in the QIIME2 [97] (version 2020.2) pipeline with recommended parameters to obtain amplicon sequence variants (ASVs). To minimize the effects of sequencing depth, the number of reads from each sample was rarefied to 23834 for comparing all samples at the same sequencing level. The taxonomy of each $16 \mathrm{~S}$ rDNA sequence was aligned against the bacteria database of SILVA [98]. QIIME2 was also used for the analysis of alpha diversity and beta diversity. 


\section{Statistical analysis and visualization}

591 Cladograms of the Sow Milk Bacterial Collection were visualized with GraPhlAn 592 v.0.9.7 [99], and the scripts were reused from a previously published paper [100]. SPSS

593 software (version 19.0; IBM Corp., Chicago, IL, USA) was used to evaluate piglet 594 experiment results with one-way analysis of variance and Duncan's multiple 595 comparison test to determine the statistical significance of the differences among 596 treatment groups. Different letters in a same graph indicate significant statistical 597 differences $(p<0.05)$.

598

599

600

601

602

603

604

605

606

607

608

609

\section{Accession codes}

The numbers and GenBank IDs of 16S rRNA genes of all smBC isolates are listed in Suppl. Data s2. The Whole Genome Shotgun project has been deposited at DDBJ/ENA/GenBank under the accession JAEMVT000000000.

\section{Supplementary information}

Additional file 1: Figure S1. The maximum-likelihood tree of the bacterial taxa. The 271 bacterial taxa were clustered based on 16S rDNA gene identity with a cut-off value of $99 \%$ for different taxa in the CD-HIT analysis. The closely related neighboring species of each taxon are listed next to the taxon numbers of the sow milk microbiota (SMM). The suspected new species are indicated in blue, and the species in the dairy products are indicated in red dots. Supplementary Data S1. The 16S rRNA sequences and classification of all isolates in smBC 
Additional file 2: Figure S2. Light microscopy images obtained from $P$. pentosaceus

611

612

613

614

615

616

617

618

619

620

621

622

623

624

625

626

627

628

629

630

strains using a 63x oil immersion objective. Supplementary Data S2. The alignment of 1240 16S rRNA sequences against the Silva version132 16S rRNA database, NCBI nucleotide collection (nr/nt) database and DAIRYdb database using BLASTN

Additional file 3: Figure S3. The antimicrobial activity of SMM914. (a) The pH of SMM914 products were determined via test papers. (b) Inhibitory effects of SMM914 against $S$. aureus and ETEC. The circular wells were filled with different products of SMM914, including MRS medium (1\&2) and the cell-free supernatant without any treatment (3\&4), with 15 min heat inactivation (5\&6), with $\mathrm{pH}$ adjustment using $\mathrm{NaOH}$ (7\&8) or with $1 \mathrm{mg} / \mathrm{mL}$ protease $\mathrm{K}$ treatment (9\&10). The substances $(1,3,5,7$ and 9$)$ were obtained from the fermentation broth after cultivation under anaerobic condition, while others $(2,4,6,8$ and 10$)$ were obtained from culture under aerobic condition.

Figure S4. Growth curves and pH value curve of SMM914 after anaerobic and aerobic fermentation. (a) The optical density at $600 \mathrm{~nm}\left(\mathrm{OD}_{600}\right)$ was measured from a starting $\mathrm{OD}_{600}$ of 0.085 . (b) The $\mathrm{pH}$ value was recorded with a $\mathrm{pH}$ meter. Data are the mean \pm s.e.m, $n=3$.

Additional file 4: Figure S5. The annotation and comparison of $P$. pentosaceus SMM914 genome. (a) The distribution of predicted CDSs of SMM914 in different categories of metabolic function by online software RAST. (b) A full genome comparison analysis of $P$. pentosaceus SMM914 with other $P$. pentosaceus strains, including Pediococcus pentosaceus SRCM100194, Pediococcus pentosaceus 
Data S3. Genes of SMM914 and predicted proteins by Pfam protein database

Additional file 5: Figure S6. Effects of SMM914 on (a) growth performance and (b) organ relative weight. Data are the mean \pm s.e.m. Statistical analysis was conducted by using one-way ANOVA. Data not sharing the same letter in each point were significantly different $(p<0.05)$. Figure S7. Effects of SMM914 on serum biochemical parameters. Data are the mean \pm s.e.m $(n=7)$. One-way ANOVA with adjustment for multiple comparisons was conducted. ${ }^{\mathrm{a}, \mathrm{b}}$ Within a variable, values with different superscripts differ $(p<0.05)$.

Additional file 6: Figure S9. (a) Alpha diversity comparisons of the gut microbiomes including Ace, Chao, Sobs index, Shannon index, Shannoneven index, Simpson index, Simpsoneven index and PD whole tree index which were analyzed using KruskalWallis $\mathrm{H}$ test and Tukey-Kramer post hoc test with $95 \%$ confidence level. Data are the mean \pm s.d $(n=7)$. $n s$, not significant differences. (b) Rarefaction curves for Shannon indices at genus level in the colon contents. Figure S10. (a) Linear discriminant analysis effect size 10 was set up with 0.05 as the alpha value for Wilcoxon test and then selected with a threshold value of 4 from phylum to genus level. (b)

Differences in relative abundance of the important and variable genera in colonic 
microbiota among three group. Each data represented the mean and SEM of relative

comparison test to determine the statistical. Different letters in the same graph indicate significant statistical differences $(p<0.05)$. Figure S11 (a) Bar charts of relative abundance at the family level in the control and treated groups obtained by sequencing group and control. Significantly different domains were tested by Wilcoxon rank-sum test. $* p<0.05 ; * * p<0.01$

\section{Abbreviations}

SPI: Sow and Piglet Integration; Nrf2: nuclear factor-E2-related factor 2; Keep1 :

Kelch-like ECH-associated protein 1; NQO-1: NADPH quinineoxidoreductase-1; HO-

1: hemeoxygenase-1; CAT: catalase; MDA: Malondialdehyde; GSH-Px: glutathione peroxidase; SOD: superoxide dismutase; LAB: lactic acid bacteria； SMM: sow milk microbiota.

\section{Acknowledgements}

667 We are grateful to Jun Hu and Liyuan Cai from Huazhong Agricultural University for their constructive suggestions. We also thank Zhuomin Han for editorial assistance.

\section{Funding}

670 This work was supported by the National Natural Science Foundation of China 671 (31700004), 2019 CAST Outstanding Chinese and Foreign Youth Exchange Program 
Research Learning and Innovative Experiment Project (S201910542041), Hunan Provincial Science and Technology Department (2019RS5001), Natural Science Foundation of Hunan Province (2019JJ50369) Construction of Innovative Provinces in Hunan Province (2019RS3022) and the National Students Platform for Innovation and Entrepreneurship Training Program (201810542034, 2020056).

Authors' contributions

JY and YLY contributed in conceiving the experiments. LLW designed and performed most experiments. XLZ, QYH, YX and CML collected the milk samples. THC, YNQ and YWC conducted antimicrobial activity experiment. QHL, YWC, QYW, and JLH performed paraquat resistance assays. CML, YCD, LW, PH, PL and TYY contributed to piglet-feeding trial. YXL, GHZ, JZL, HHC, PH, CC and HSY contributed the data analysis. ZZZ, JZL, HSY, CNW and QYW revised the work. JY and LLW drafted the work. All authors read and approved the manuscript.

\section{Availability of data and materials}

687

The raw sequence data reported in this paper have been deposited (PRJCA003003) in the Genome Sequence Archive in the BIG Data Center, Chinese Academy of Sciences, under accession code CRA002995 for bacterial 16S rRNA gene sequencing data, which are publicly accessible at http://bigd.big.ac.cn/gsa.

Ethics approval and consent to participate

692 Not applicable 
Not applicable

\section{Competing interests}

No conflict of interest exists in the submission of this manuscript, and the manuscript

697 has been approved by all authors for publication. The authors declare that they have no

698 competing interests.

\section{Author details}

$700 \quad{ }^{1}$ Laboratory of Animal Nutrition and Human Health, Hunan Provincial Key Laboratory 701 of Animal Intestinal Function and Regulation, Hunan International Joint Laboratory of 702 Animal Intestinal Ecology and Health, College of Life Sciences, Hunan Normal 703 University, Changsha, China.

$704{ }^{2}$ Institute of Subtropical Agriculture, Chinese Academy of Sciences, Changsha, China

$705{ }^{3}$ The National and Local Joint Engineering Laboratory of Animal Peptide Drug

706 Development, College of Life Sciences, Hunan Normal University, Changsha, China

$707{ }^{4}$ State Key Laboratory of Plant Genomics, Institute of Genetics and Developmental

708 Biology, Chinese Academy of Sciences, Beijing, China

$709{ }^{5}$ College of Animal Science and Technology, Hunan Agricultural University,

710 Changsha, Hunan, China.

$711{ }^{6}$ Shandong Yihe Feed Co.,Ltd. Yantai Hi-tech Industrial Development Zone, Yantai, 712 Shandong, China. 


\section{References}

1. Stewart CJ, Ajami NJ, O'Brien JL, Hutchinson DS, Smith DP, Wong MC, et al. Temporal development of the gut microbiome in early childhood from the TEDDY study. Nature. 2018;562(7728):583-8.

2. Breastfeeding and the Use of Human Milk. 1997;100(6):1035-9.

3. Li Z, Wright AG, Yang Y, Si H, Li G. Unique Bacteria Community Composition and Cooccurrence in the Milk of Different Ruminants. Scientific reports. 2017;7:40950.

4. Fitzstevens JL, Smith KC, Hagadorn JI, Caimano MJ, Matson AP, Brownell EA. Systematic Review of the Human Milk Microbiota. Nutr Clin Pract. 2017;32(3):354-64.

5. Gil-Campos M, López M, Rodriguez-Benítez MV, Romero J, Roncero I, Linares MD, et al. Lactobacillus fermentum CECT 5716 is safe and well tolerated in infants of 1-6 months of age: a randomized controlled trial. Pharmacological research. 2012;65(2):231-8.

6. Eckert R, Mucha A, Koska M, Szulc T. Relationships Between Chemical Composition of Colostrum and Milk and Rearing Performance of Piglets During a 21-Day Lactation. Annals of Animal 2013;13(4):771-81.

7. Meckel KR, Kiraly DD. Maternal microbes support fetal brain wiring. Nature. 2020;586(7828):203-5.

8. Fernandez L, Langa S, Martin V, Maldonado A, Jimenez E, Martin R, et al. The human milk microbiota: origin and potential roles in health and disease. Pharmacological research. 2013;69(1):1-10.

9. Chen W, Mi J, Lv N, Gao J, Cheng J, Wu R, et al. Lactation Stage-Dependency of the Sow Milk Microbiota. Front Microbiol. 2018;9:945.

10. Odamaki T, Bottacini F, Kato K, Mitsuyama E, Yoshida K, Horigome A, et al. Genomic diversity and distribution of Bifidobacterium longum subsp. longum across the human lifespan. Scientific reports. 2018;8(1):85.

11. Solieri L, Rutella GS, Tagliazucchi D. Impact of non-starter lactobacilli on release of peptides with angiotensin-converting enzyme inhibitory and antioxidant activities during bovine milk fermentation. Food microbiology. 2015;51:108-16.

12. Chen T, Wang L, Li Q, Long Y, Lin Y, Yin J, et al. Functional probiotics of lactic acid bacteria from Hu sheep milk. BMC microbiology. 2020;20(1):228.

13. Altuntas EG, Ayhan K, Peker S, Ayhan B, Demiralp DO. Purification and mass spectrometry based characterization of a pediocin produced by Pediococcus acidilactici 13. Mol Biol Rep. 2014;41(10):6879-85.

14. Anastasiadou S, Papagianni M, Filiousis G, Ambrosiadis I, Koidis P. Growth and metabolism of a meat isolated strain of Pediococcus pentosaceus in submerged fermentation: Purification, characterization and properties of the produced pediocin SM-1. Enzyme and Microbial Technology. 2008;43(6):448-54.

15. Porto MC, Kuniyoshi TM, Azevedo PO, Vitolo M, Oliveira RP. Pediococcus spp.: An important genus of lactic acid bacteria and pediocin producers. Biotechnology advances. 2017;35(3):361-74. 
16. Wang X, Lu H, Feng Z, Cao J, Fang C, Xu X, et al. Development of Human Breast Milk Microbiota-Associated Mice as a Method to Identify Breast Milk Bacteria Capable of Colonizing Gut. Frontiers in Microbiology. 2017;8(1242).

17. Liu H, Hou C, Li N, Zhang X, Zhang G, Yang F, et al. Microbial and metabolic alterations in gut microbiota of sows during pregnancy and lactation. FASEB J. 2019;33(3):4490501.

18. Carroll L, Osman M, Davies DP, McNeish AS. Bacteriological criteria for feeding raw breast-milk to babies on neonatal units. Lancet. 1979;2(8145):732-3.

19. West PA, Hewitt JH, Murphy OM. Influence of methods of collection and storage on the bacteriology of human milk. J Appl Bacteriol. 1979;46(2):269-77.

20. Cacho NT, Harrison NA, Parker LA, Padgett KA, Lemas DJ, Marcial GE, et al. Personalization of the Microbiota of Donor Human Milk with Mother's Own Milk. Front Microbiol. 2017;8:1470.

21. Dubreuil JD, Isaacson RE, Schifferli DM. Animal Enterotoxigenic Escherichia coli. EcoSal Plus. 2016;7(1).

22. Barton MD. Antibiotic use in animal feed and its impact on human healt. Nutr Res Rev. 2000;13(2):279-99.

23. Lee JW, Choi YC, Kim R, Lee SK. Multiwall Carbon Nanotube-Induced Apoptosis and Antioxidant Gene Expression in the Gills, Liver, and Intestine of Oryzias latipes. Biomed Res Int. 2015;2015:485343.

24. Moxley RA, Francis DH, Tamura M, Marx DB, Santiago-Mateo K, Zhao M. Efficacy of Urtoxazumab (TMA-15 Humanized Monoclonal Antibody Specific for Shiga Toxin 2) Against Post-Diarrheal Neurological Sequelae Caused by Escherichia coli O157:H7 Infection in the Neonatal Gnotobiotic Piglet Model. Toxins (Basel). 2017;9(2).

25. Wei H, Shen J, Pang X, Ding D, Zhang Y, Zhang B, et al. Fatal infection in human floraassociated piglets caused by the opportunistic pathogen Klebsiella pneumoniae from an apparently healthy human donor. J Vet Med Sci. 2008;70(7):715-7.

26. Igbinosa $\mathrm{IH}$, Igbinosa $\mathrm{EO}, \mathrm{Okoh}$ Al. Antibiogram characterization and putative virulence genes in Aeromonas species isolated from pig fecal samples. Environ Sci Pollut Res Int. 2016;23(12):12199-205.

27. Kock R, Cuny C. [Multidrug-resistant bacteria in animals and humans]. Med Klin Intensivmed Notfmed. 2020;115(3):189-97.

28. Zheng $\mathrm{L}, \mathrm{Hu} \mathrm{Y}, \mathrm{He} \mathrm{X}$, Zhao $\mathrm{Y}, \mathrm{Xu} \mathrm{H}$. Isolation of swine-derived Lactobacillus plantarum and its synergistic antimicrobial and health-promoting properties with $\mathrm{ZnO}$ nanoparticles. J Appl Microbiol. 2020.

29. V R, Kumar R, R.R.B S, Kaushik J, Mann B. Comparative evaluation of selected strains of lactobacilli for the development of antioxidant activity in milk. Dairy Science and Technology. 2011;92.

30. Nie $\mathrm{Y}, \mathrm{Hu}$ J, Hou Q, Zheng $\mathrm{W}$, Zhang $\mathrm{X}$, Yang $\mathrm{T}$, et al. Lactobacillus frumenti improves antioxidant capacity via nitric oxide synthase 1 in intestinal epithelial cells. Faseb j. 2019;33(10):10705-16. 
31. Patel PH, Pénalva C, Kardorff M, Roca M, Pavlović B, Thiel A, et al. Damage sensing by a Nox-Ask1-MKK3-p38 signaling pathway mediates regeneration in the adult Drosophila midgut. Nature communications. 2019;10(1):4365.

32. Darby TM, Owens JA, Saeedi BJ, Luo L, Matthews JD, Robinson BS, et al. Lactococcus Lactis Subsp. cremoris Is an Efficacious Beneficial Bacterium that Limits Tissue Injury in the Intestine. iScience. 2019;12:356-67.

33. Papadopoulos GA, Poutahidis T, Tallarico N, Hardas A, Teliousis K, Arsenos G, et al. Dietary supplementation of encapsulated organic acids enhances performance and modulates immune regulation and morphology of jejunal mucosa in piglets. Research in veterinary science. 2017;115:174-82.

34. Serata M, lino T, Yasuda E, Sako T. Roles of thioredoxin and thioredoxin reductase in the resistance to oxidative stress in Lactobacillus casei. Microbiology. 2012;158(Pt_4):953-62.

35. Zhang L, Liu C, Li D, Zhao Y, Zhang X, Zeng X, et al. Antioxidant activity of an exopolysaccharide isolated from Lactobacillus plantarum C88. Int J Biol Macromol. 2013:54:270-5.

36. Zhang B, Li S, Men J, Peng C, Shao H, Zhang Z. Long-term exposure to crotonaldehyde causes heart and kidney dysfunction through induction of inflammatory and oxidative damage in male Wistar rats. Toxicology Mechanisms and Methods. 2019;29(4):263 - 75.

37. Lushchak VI, Bagnyukova TV, Lushchak OV, Storey JM, Storey KB. Hypoxia and recovery perturb free radical processes and antioxidant potential in common carp (Cyprinus carpio) tissues. The International Journal of Biochemistry \& Cell Biology. 2005;37(6):1319-30.

38. Mutanen A, Lohi J, Sorsa T, Jalanko H, Pakarinen MP. Features of liver tissue remodeling in intestinal failure during and after weaning off parenteral nutrition. Surgery. 2016;160(3):632-42.

39. Lev-Cohain N, Sapir G, Harris T, Azar A, Gamliel A, Nardi-Schreiber A, et al. Real-time ALT and LDH activities determined in viable precision-cut mouse liver slices using hyperpolarized [1-(13) C]pyruvate-Implications for studies on biopsied liver tissues. NMR Biomed. 2019;32(2):e4043.

40. Elhelaly AE, AlBasher G, Alfarraj S, Almeer R, Bahbah El, Fouda MMA, et al. Protective effects of hesperidin and diosmin against acrylamide-induced liver, kidney, and brain oxidative damage in rats. Environ Sci Pollut Res Int. 2019;26(34):35151-62.

41. Deng H, Kerppola TK. Regulation of Drosophila metamorphosis by xenobiotic response regulators. PLoS Genet. 2013;9(2):e1003263.

42. Chen-Roetling J, Regan RF. Targeting the Nrf2-Heme Oxygenase-1 Axis after Intracerebral Hemorrhage. Curr Pharm Des. 2017;23(15):2226-37.

43. Chen J, Xie H, Chen D, Yu B, Mao X, Zheng P, et al. Chlorogenic Acid Improves Intestinal Development via Suppressing Mucosa Inflammation and Cell Apoptosis in Weaned Pigs. ACS Omega. 2018;3(2):2211-9.

44. Pan D, Mei X. Antioxidant activity of an exopolysaccharide purified from Lactococcus lactis subsp. lactis 12. Carbohydrate Polymers. 2010;80(3):908-14. 
45. Jiao N, Wu Z, Ji Y, Wang B, Dai Z, Wu G. L-Glutamate Enhances Barrier and Antioxidative Functions in Intestinal Porcine Epithelial Cells. J Nutr. 2015;145(10):2258-64.

46. Lopes IS, Oliveira ICM, Capibaribe VCC, Valentim JT, da Silva DMA, de Souza AG, et al. Riparin II ameliorates corticosterone-induced depressive-like behavior in mice: Role of antioxidant and neurotrophic mechanisms. Neurochem Int. 2018;120:33-42.

47. O'Mahony SM, Hyland NP, Dinan TG, Cryan JF. Maternal separation as a model of braingut axis dysfunction. Psychopharmacology (Berl). 2011;214(1):71-88.

48. Kember RL, Dempster EL, Lee TH, Schalkwyk LC, Mill J, Fernandes C. Maternal separation is associated with strain-specific responses to stress and epigenetic alterations to $\mathrm{Nr} 3 \mathrm{c} 1$, Avp, and Nr4a1 in mouse. Brain Behav. 2012;2(4):455-67.

49. Davis MY, Zhang H, Brannan LE, Carman RJ, Boone JH. Rapid change of fecal microbiome and disappearance of Clostridium difficile in a colonized infant after transition from breast milk to cow milk. Microbiome. 2016;4(1):53.

50. Leung C, Rivera L, Furness JB, Angus PW. The role of the gut microbiota in NAFLD. Nat Rev Gastroenterol Hepatol. 2016;13(7):412-25.

51. Napolitano A, Miller S, Nicholls A, Baker D, Horn S, Thomas E, et al. Novel Gut-Based Pharmacology of Metformin in Patients with Type 2 Diabetes Mellitus. PloS one. 2014;9:e100778.

52. Wu W, Xiao Z, An W, Dong Y, Zhang B. Dietary sodium butyrate improves intestinal development and function by modulating the microbial community in broilers. PLoS One. 2018;13(5):e0197762.

53. Chen Y, Guo J, Qian G, Fang D, Shi D, Guo L, et al. Gut dysbiosis in acute-on-chronic liver failure and its predictive value for mortality. J Gastroenterol Hepatol. 2015;30(9):1429-37.

54. Buchet A, Belloc C, Leblanc-Maridor M, Merlot E. Effects of age and weaning conditions on blood indicators of oxidative status in pigs. PLoS One. 2017;12(5):e0178487.

55. Smith A, Stalder K, Serenius T, Serenius, Msc T, Baas T, et al. Effect of weaning age on nursery pig and sow reproductive performance. Journal of Swine Health and Production. 2008;16:131-7.

56. Adetoye A, Pinloche E, Adeniyi BA, Ayeni FA. Characterization and anti-salmonella activities of lactic acid bacteria isolated from cattle faeces. BMC microbiology. 2018;18(1):96.

57. Zheng W, Zhao W, Wu M, Song X, Caro F, Sun X, et al. Microbiota-targeted maternal antibodies protect neonates from enteric infection. Nature. 2020;577(7791):543-8.

58. Difilippo E, Pan F, Logtenberg M, Willems RH, Braber S, Fink-Gremmels J, et al. In Vitro Fermentation of Porcine Milk Oligosaccharides and Galacto-oligosaccharides Using Piglet Fecal Inoculum. J Agric Food Chem. 2016;64(10):2127-33.

59. Fernandez L, Ruiz L, Jara J, Orgaz B, Rodriguez JM. Strategies for the Preservation, Restoration and Modulation of the Human Milk Microbiota. Implications for Human Milk Banks and Neonatal Intensive Care Units. Front Microbiol. 2018;9:2676. 
60. Lagier JC, Khelaifia S, Alou MT, Ndongo S, Dione N, Hugon P, et al. Culture of previously uncultured members of the human gut microbiota by culturomics. Nat Microbiol. 2016;1:16203.

61. Lv L-X, Hu X-J, Qian G-R, Lu H-F, Zheng B-W, Jiang L, et al. Administration of Lactobacillus salivarius LI01 or Pediococcus pentosaceus LI05 improves acute liver injury induced by D-galactosamine in rats. Applied microbiology and biotechnology. 2014;98.

62. Itoh K, Tong Kl, Yamamoto M. Molecular mechanism activating nrf2-keap1 pathway in regulation of adaptive response to electrophiles. Free Radical Biology and Medicine. 2004;36(10):1208-13.

63. Schwab CG, Bozak CK, Whitehouse NL, Olson VM. Amino acid limitation and flow to the duodenum at four stages of lactation. 2. Extent of lysine limitation. J Dairy Sci. 1992;75(12):3503-18.

64. Zeitz JO, Kaltenböck S, Most E, Eder K. Effects of L-methionine on performance, gut morphology and antioxidant status in gut and liver of piglets in relation to DLmethionine. Journal of animal physiology and animal nutrition. 2019;103(1):242-50.

65. Zhou YF, Zhou Z, Batistel F, Martinez-Cortés I, Pate RT, Luchini DL, et al. Methionine and choline supply alter transmethylation, transsulfuration, and cytidine 5 '-diphosphocholine pathways to different extents in isolated primary liver cells from dairy cows. J Dairy Sci. 2018;101(12):11384-95.

66. Swennen Q, Geraert PA, Mercier Y, Everaert N, Stinckens A, Willemsen H, et al. Effects of dietary protein content and 2-hydroxy-4-methylthiobutanoic acid or DL-methionine supplementation on performance and oxidative status of broiler chickens. The British journal of nutrition. 2011;106(12):1845-54.

67. Bos C, Stoll B, Fouillet H, Gaudichon C, Guan X, Grusak MA, et al. Postprandial intestinal and whole body nitrogen kinetics and distribution in piglets fed a single meal. Am J Physiol Endocrinol Metab. 2005;288(2):E436-46.

68. Gamarra Y, Santiago FC, Molina-Lopez J, Castano J, Herrera-Quintana L, Dominguez A, et al. Pyroglutamic acidosis by glutathione regeneration blockage in critical patients with septic shock. Crit Care. 2019;23(1):162.

69. Zeisel SH. Dietary choline deficiency causes DNA strand breaks and alters epigenetic marks on DNA and histones. Mutat Res. 2012;733(1-2):34-8.

70. Zhou Z, Vailati-Riboni M, Luchini DN, Loor J]. Methionine and Choline Supply during the Periparturient Period Alter Plasma Amino Acid and One-Carbon Metabolism Profiles to Various Extents: Potential Role in Hepatic Metabolism and Antioxidant Status. Nutrients. 2016;9(1).

71. Guo WX, Pye QN, Williamson KS, Stewart CA, Hensley KL, Kotake Y, et al. Reactive oxygen species in choline deficiency-induced apoptosis in rat hepatocytes. Free Radic Biol Med. 2004;37(7):1081-9.

72. Tabassum S, Haider S, Ahmad S, Madiha S, Parveen T. Chronic choline supplementation improves cognitive and motor performance via modulating oxidative and neurochemical status in rats. Pharmacol Biochem Behav. 2017;159:90-9. 
73. Coleman DN, Vailati-Riboni M, Elolimy AA, Cardoso FC, Rodriguez-Zas SL, Miura M, et al. Hepatic betaine-homocysteine methyltransferase and methionine synthase activity and intermediates of the methionine cycle are altered by choline supply during negative energy balance in Holstein cows. J Dairy Sci. 2019;102(9):8305-18.

74. Wu P, Liu Y, Jiang WD, Jiang J, Zhao J, Zhang YA, et al. A Comparative Study on Antioxidant System in Fish Hepatopancreas and Intestine Affected by Choline Deficiency: Different Change Patterns of Varied Antioxidant Enzyme Genes and Nrf2 Signaling Factors. PLoS One. 2017;12(1):e0169888.

75. Li B, Li W, Ahmad H, Zhang L, Wang C, Wang T. Effects of Choline on Meat Quality and Intramuscular Fat in Intrauterine Growth Retardation Pigs. PLoS One. 2015;10(6):e0129109.

76. Yamada S, Kawaguchi H, Yamada T, Guo X, Matsuo K, Hamada T, et al. Cholic Acid Enhances Visceral Adiposity, Atherosclerosis and Nonalcoholic Fatty Liver Disease in Microminipigs. J Atheroscler Thromb. 2017;24(11):1150-66.

77. Carrier A. Metabolic Syndrome and Oxidative Stress: A Complex Relationship. Antioxid Redox Signal. 2017;26(9):429-31.

78. Sommerfeld A, Reinehr R, Haussinger D. Bile acid-induced epidermal growth factor receptor activation in quiescent rat hepatic stellate cells can trigger both proliferation and apoptosis. J Biol Chem. 2009;284(33):22173-83.

79. Becker BA, Nienaber JA, Christenson RK, Manak RC, DeShazer JA, Hahn GL. Peripheral concentrations of cortisol as an indicator of stress in the pig. American journal of veterinary research. 1985;46(5):1034-8.

80. Du X, Pang TY. Is Dysregulation of the HPA-Axis a Core Pathophysiology Mediating CoMorbid Depression in Neurodegenerative Diseases? Frontiers in psychiatry. 2015;6:32.

81. Kulak A, Steullet P, Cabungcal JH, Werge T, Ingason A, Cuenod M, et al. Redox dysregulation in the pathophysiology of schizophrenia and bipolar disorder: insights from animal models. Antioxid Redox Signal. 2013;18(12):1428-43.

82. Lin X, Xia Y, Wang G, Xiong Z, Zhang H, Lai F, et al. Lactobacillus plantarum AR501 Alleviates the Oxidative Stress of D-Galactose-Induced Aging Mice Liver by Upregulation of Nrf2-Mediated Antioxidant Enzyme Expression. J Food Sci. 2018;83(7):1990-8.

83. Jiang T, Xing X, Zhang L, Liu Z, Zhao J, Liu X. Chitosan Oligosaccharides Show Protective Effects in Coronary Heart Disease by Improving Antioxidant Capacity via the Increase in Intestinal Probiotics. Oxid Med Cell Longev. 2019;2019:7658052.

84. Wu CH, Ko JL, Liao JM, Huang SS, Lin MY, Lee LH, et al. D-methionine alleviates cisplatin-induced mucositis by restoring the gut microbiota structure and improving intestinal inflammation. Ther Adv Med Oncol. 2019;11:1758835918821021.

85. Ley RE, Bäckhed F, Turnbaugh P, Lozupone CA, Knight RD, Gordon Jl. Obesity alters gut microbial ecology. Proceedings of the National Academy of Sciences of the United States of America. 2005;102(31):11070-5. 
86. Wang K, Jin X, Li Q, Sawaya A, Le Leu RK, Conlon MA, et al. Propolis from Different Geographic Origins Decreases Intestinal Inflammation and Bacteroides spp. Populations in a Model of DSS-Induced Colitis. Mol Nutr Food Res. 2018;62(17):e1800080.

87. Takano S, Pawlowska BJ, Gudelj I, Yomo T, Tsuru S. Density-Dependent Recycling Promotes the Long-Term Survival of Bacterial Populations during Periods of Starvation. mBio. 2017;8(1).

88. Wang D, Liu W, Ren Y, De L, Zhang D, Yang Y, et al. Isolation and Identification of Lactic Acid Bacteria from Traditional Dairy Products in Baotou and Bayannur of Midwestern Inner Mongolia and q-PCR Analysis of Predominant Species. Korean J Food Sci Anim Resour. 2016;36(4):499-507.

89. Grill JP, Cayuela C, Antoine JM, Schneider F. Effects of Lactobacillus amylovorus and Bifidobacterium breve on cholesterol. Lett Appl Microbiol. 2000;31(2):154-6.

90. Limkhuansuwan V, Chaiprasert P. Decolorization of molasses melanoidins and palm oil mill effluent phenolic compounds by fermentative lactic acid bacteria. J Environ Sci (China). 2010;22(8):1209-17.

91. Hu J, Ma L, Nie Y, Chen J, Zheng W, Wang X, et al. A Microbiota-Derived Bacteriocin Targets the Host to Confer Diarrhea Resistance in Early-Weaned Piglets. Cell host \& microbe. 2018;24(6):817-32.e8.

92. Janda JM, Abbott SL. 16S rRNA gene sequencing for bacterial identification in the diagnostic laboratory: pluses, perils, and pitfalls. J Clin Microbiol. 2007;45(9):2761-4.

93. Ponce AG, Moreira MR, del Valle CE, Roura SI. Preliminary characterization of bacteriocin-like substances from lactic acid bacteria isolated from organic leafy vegetables. LWT - Food Science and Technology. 2008;41(3):432-41.

94. Magoč T, Salzberg SL. FLASH: fast length adjustment of short reads to improve genome assemblies. Bioinformatics (Oxford, England). 2011;27(21):2957-63.

95. Chen S, Zhou Y, Chen Y, Gu J. fastp: an ultra-fast all-in-one FASTQ preprocessor.

96. Callahan BJ, McMurdie PJ, Rosen MJ, Han AW, Johnson AJ, Holmes SP. DADA2: Highresolution sample inference from Illumina amplicon data. Nature methods. 2016;13(7):581-3.

97. Bolyen E, Rideout JR, Dillon MR, Bokulich NA, Abnet CC, Al-Ghalith GA, et al. Reproducible, interactive, scalable and extensible microbiome data science using QIIME 2. Nature biotechnology. 2019;37(8):852-7.

98. Quast C, Pruesse E, Yilmaz P, Gerken J, Schweer T, Yarza P, et al. The SILVA ribosomal RNA gene database project: improved data processing and web -based tools. Nucleic acids research. 2013;41(Database issue):D590-6.

99. Asnicar F, Weingart G, Tickle TL, Huttenhower C, Segata N. Compact graphical representation of phylogenetic data and metadata with GraPhIAn. PeerJ. 2015;3:e1029.

100. Zhang J, Liu YX, Zhang N, Hu B, Jin T, Xu H, et al. NRT1.1B is associated with root microbiota composition and nitrogen use in field-grown rice. Nature biotechnology. 2019;37(6):676-84. 


\section{Figure Legends}

Figure 1. The workflow for large-scale bacterial cultivation from sow milk and the 1001 characterization of probiotic functions.

Figure 2. The Sow Milk Bacterial Collection. The inner circles depict taxonomic assignments of bacteria for the indicated phyla, classes, orders, families, genera, and species that were identified. The species names are labeled, and the numbers of different taxa within each species are provided at the nodes. P. pentosaceus is indicated in red. The probability (\%) of suspected new species and known species identified from the dairy products is shown in the outer ring with the green or yellow heat map, respectively. The outermost bar plots represent the numbers of isolates (log2 transformed) of each species.

Figure 3. Screening of potential probiotics prior to a piglet feeding experiment. (a) Antagonistic activity against pathogens in vitro. (b) Graphical representation of experiments conducted to assess the survival rate. (c) $P$. pentosaceus confers protective effects upon Drosophila. Statistical differences were calculated by the log-rank test.

Figure 4. Effects of the oral administration of SMM914 on the antioxidation capacity and activation of the cytoprotective Nrf2 pathway in piglets. (a) Experimental outline in piglets $(n=18)$. Piglets were kept for 10 days after birth for adaptation. Oral administration of SMM914 was performed on days 10, 12, 14, 16 and 18 at low or high 
1019

1020

1021

1022

1023

1024

1025

1026

1027

1028

1029

1030

1031

1032

1033

1034

1035

1036

1037

1038

slaughter for sample collection. (b) Alanine amiotransferase (ALT) and lactate dehydrogenase (LDH) were significantly decreased in piglets subjected to SMM914. (c-d) Bar graph of western blotting showing Nrf2 protein expression levels in the liver normalized to PCNA expression in the nucleus. (e-f) Western blotting analysis of antioxidant protein (Keap1, NQO-1, HO-1, SOD1 and CAT) levels in the liver and bar graph of data showing their protein expression normalized to $\beta$-actin expression in hepatic cells $(n=6)$. (g) The enzyme activities of GSH-Px, SOD, CAT and MDA were measured in liver lysates. Values of the bars stand for significant differences using oneway ANOVA followed by Duncan's multiple comparisons at $p<0.05$. Data are the mean \pm s.e.m, $n=7$.

Figure 5. The metabolic profile of plasma. Score plots of the PLS-DA analysis for the plasma metabolome (a) showing the scatter between the control and low-dose groups. (b) Scatter between the control and high-dose groups. (c) The integrative metabolism pathway according to the KEGG pathway database. Compared with the control group, the blue metabolites represent the intensities of metabolites that were downregulated, while the red metabolites represent the intensities of metabolites that were upregulated. (d-h) The perturbed metabolism pathways and metabolites in response to SMM914 treatment mainly include glutathione metabolism; cysteine and methionine metabolism; glycine, serine and threonine metabolism; and arginine and proline metabolism. Data are the mean \pm s.e.m, $n=7$. 
1039

1040

1041

1042

1043

1044

1045

1046

1047

1048

1049

1050

1051

1052

1053

1054

1055

1056

1057

1058

1059

Figure 6. Microbial composition analysis in the colon. (a) The Venn diagram for operational taxonomic units among the control group, low-dose group and high-dose group. (b) Scatterplot of principal component analysis in bacterial communities. Principal components (PCs) 1 and 2 explain $11.11 \%$ and $9.96 \%$ of the variance, respectively (permutational multivariate analysis of variance). (c) Individual (left) and averaged (right) taxon summary of bacterial genera in colon contents.

Figure 7. (a) Cladogram generated from Linear discriminant analysis (LDA) effect size (LEfSe) analysis for the most differentially abundant taxa with LDA scores above 4.

(b) Comparison of microbial genera between high dose group and control. Significantly different domains were tested by Wilcoxon rank-sum test. $* p<0.05 ; * * p<0.01$.

Figure S1. The maximum-likelihood tree of the bacterial taxa. The 271 bacterial taxa were clustered based on $16 \mathrm{~S}$ rDNA gene identity with a cut-off value of $99 \%$ for different taxa in the CD-HIT analysis. The closely related neighboring species of each taxon are listed next to the taxon numbers of the sow milk microbiota. The suspected new species are indicated in blue, and the species in the dairy products are indicated in red dots.

Figure S2. Light microscopy images obtained from $P$. pentosaceus strains using a $63 \mathrm{x}$ oil immersion objective.

Figure S3. The antimicrobial activity of SMM914. (a) The pH of SMM914 products were determined via test papers. (b) Inhibitory effects of SMM914 against S. aureus and ETEC. The circular wells were filled with different products of SMM914, including 
MRS medium (1\&2) and the cell-free supernatant without any treatment (3\&4), with 15 min heat inactivation (5\&6), with $\mathrm{pH}$ adjustment using $\mathrm{NaOH}(7 \& 8)$ or with 1 $\mathrm{mg} / \mathrm{mL}$ protease $\mathrm{K}$ treatment (9\&10). The substances (1, 3, 5, 7 and 9) were obtained 1063 from the fermentation broth after cultivation under anaerobic condition, while others $(2,4,6,8$ and 10) were obtained from culture under aerobic condition.

Figure S4. Growth curves and pH value curve of SMM914 after anaerobic and aerobic 1066 fermentation. (a) The optical density at $600 \mathrm{~nm}\left(\mathrm{OD}_{600}\right)$ was measured from a starting $\mathrm{OD}_{600}$ of 0.085 . (b) The $\mathrm{pH}$ value was recorded with a $\mathrm{pH}$ meter. Data are the mean \pm s.e.m, $n=3$.

Figure S5. The annotation and comparison of P. pentosaceus SMM914 genome. (a) The distribution of predicted CDSs of SMM914 in different categories of metabolic 1071 function by online software RAST. (b) A full genome comparison analysis of $P$. 1072 pentosaceus SMM914 with other P. pentosaceus strains, including Pediococcus 1073 pentosaceus SRCM100194, Pediococcus pentosaceus GDIAS001, Pediococcus pentosaceus SL001 and Pediococcus pentosaceus SRCM102736, visualized by BRIG 1075 software. Colors display the percentage of sequence identity based on BLASTN. Two 1076 inner rings indicate the $\mathrm{GC}$ skew and the $\mathrm{G}+\mathrm{C}$ content. The innermost circle shows 1077 the genome coordinates.

1078 Figure S6. Effects of SMM914 on (a) growth performance and (b) organ relative 1079 weight. Data are the mean \pm s.e.m. Statistical analysis was conducted by using one-way 
ANOVA. Data not sharing the same letter in each point were significantly different ( $p$ $<0.05)$.

Figure S7. Effects of SMM914 on serum biochemical parameters. Data are the mean \pm s.e.m $(n=7)$. One-way ANOVA with adjustment for multiple comparisons was conducted. ${ }^{\mathrm{a}, \mathrm{b}}$ Within a variable, values with different superscripts $\operatorname{differ}(p<0.05)$.

Figure S8. (a) In HepG2 cell and (b) IPEC-J2 cell, western blotting experiment was to determine the effect of SMM914 in MRS broth after 24h fermentation on the protein level of Nrf2. (c) The relative changes in protein intensity were analyzed with unpaired $(p<0.05, n=3)$.

Figure S9. (a) Alpha diversity comparisons of the gut microbiomes including Ace, Chao, Sobs index, Shannon index, Shannoneven index, Simpson index, Simpsoneven index and PD whole tree index which were analyzed using Kruskal-Wallis H test and Tukey-Kramer post hoc test with $95 \%$ confidence level. Data are the mean \pm s.d $(n=$ level in the colon contents.

Figure S10. (a) Linear discriminant analysis effect size 10 was set up with 0.05 as the alpha value for Wilcoxon test and then selected with a threshold value of 4 from phylum to genus level. (b) Differences in relative abundance of the important and variable 1099 genera in colonic microbiota among three group. Each data represented the mean and 

same graph indicate significant statistical differences $(p<0.05)$.

Figure S11. (a) Bar charts of relative abundance at the family level in the control and treated groups obtained by sequencing of $16 \mathrm{~S}$ rDNA V3-V4 region. (b) Comparison of 1105 microbial families between high dose group and control. Significantly different 1106 domains were tested by Wilcoxon rank-sum test. * $p<0.05$; ** $p<0.01$.

1107 Supplementary Data S1. The 16S rRNA sequences and classification of all isolates 1108 in $\mathrm{smBC}$

1109 Supplementary Data S2. The alignment of 1240 16S rRNA sequences against the 1110 Silva version132 16S rRNA database, NCBI nucleotide collection (nr/nt) database 1111 and DAIRYdb database using BLASTN

1112 Supplementary Data S3. Genes of SMM914 and predicted proteins by Pfam protein 1113 database 
Figures

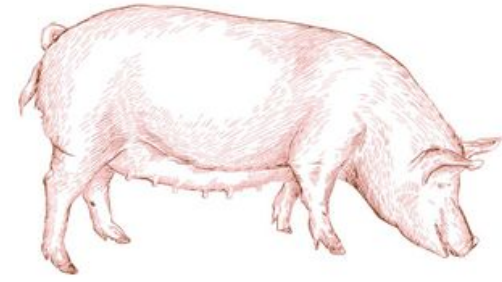

Step 1. Fresh sow milk collection
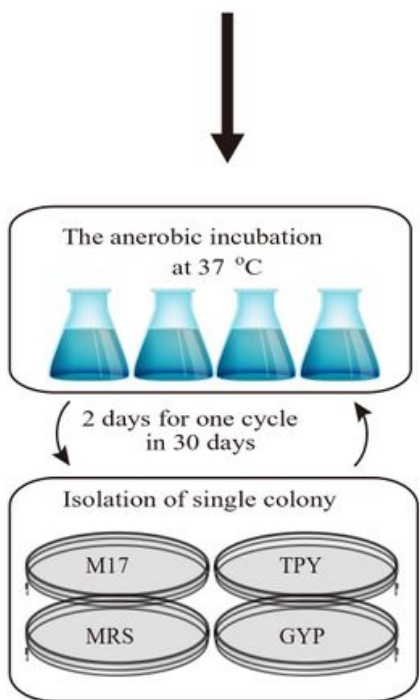

Step 2. Collection of lactic acid bacteria

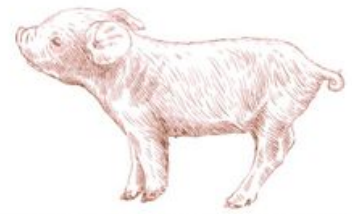

Step 7. Early weaning piglets model

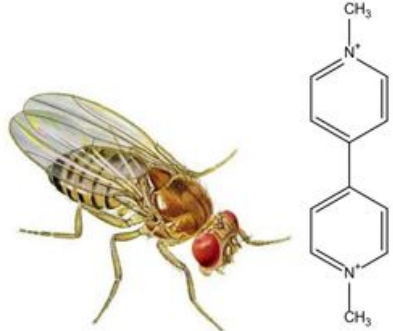

Step 6. Paraquat resistance in Drosophlia
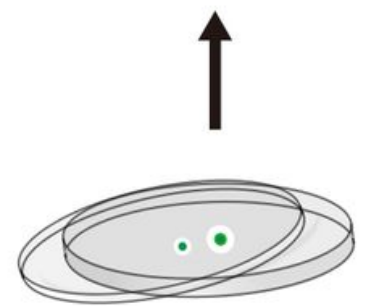

Step 5. Antagonistic activity in vitro

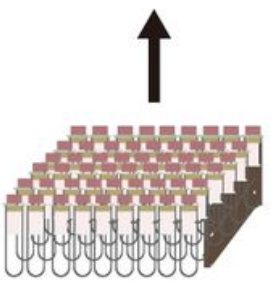

Step 4. Strain preservation

Figure 1

\section{Figure 1}

The workflow for large-scale bacterial cultivation from sow milk and the characterization of probiotic functions. 


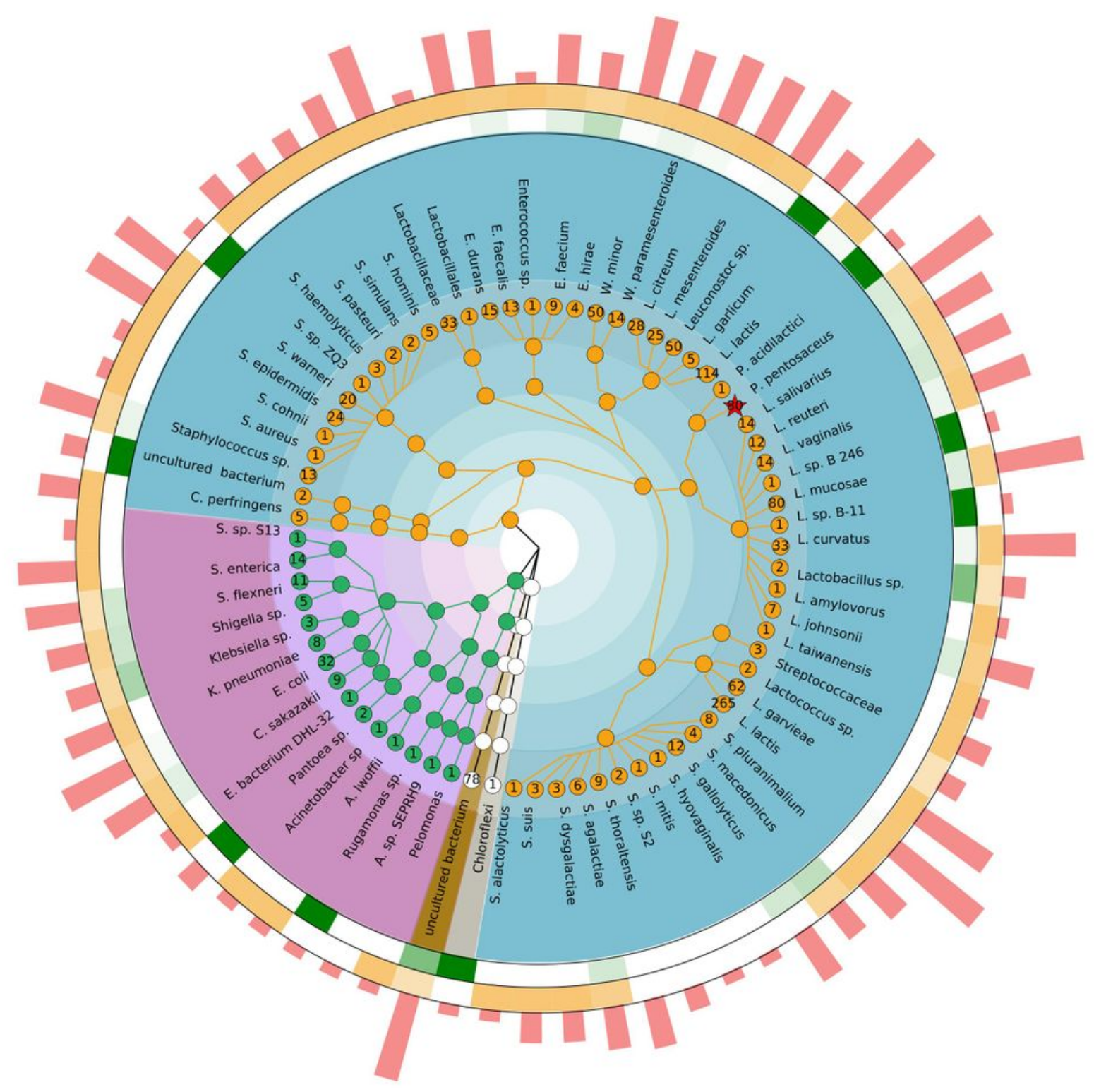

Figure 2

\section{Figure 2}

The Sow Milk Bacterial Collection. The inner circles depict taxonomic assignments of bacteria for the indicated phyla, classes, orders, families, genera, and species that were identified. The species names are labeled, and the numbers of different taxa within each species are provided at the nodes. P. pentosaceus is indicated in red. The probability (\%) of suspected new species and known species identified from the dairy products is shown in the outer ring with the green or yellow heat map, respectively. The outermost bar plots represent the numbers of isolates (log2 transformed) of each species. 
a

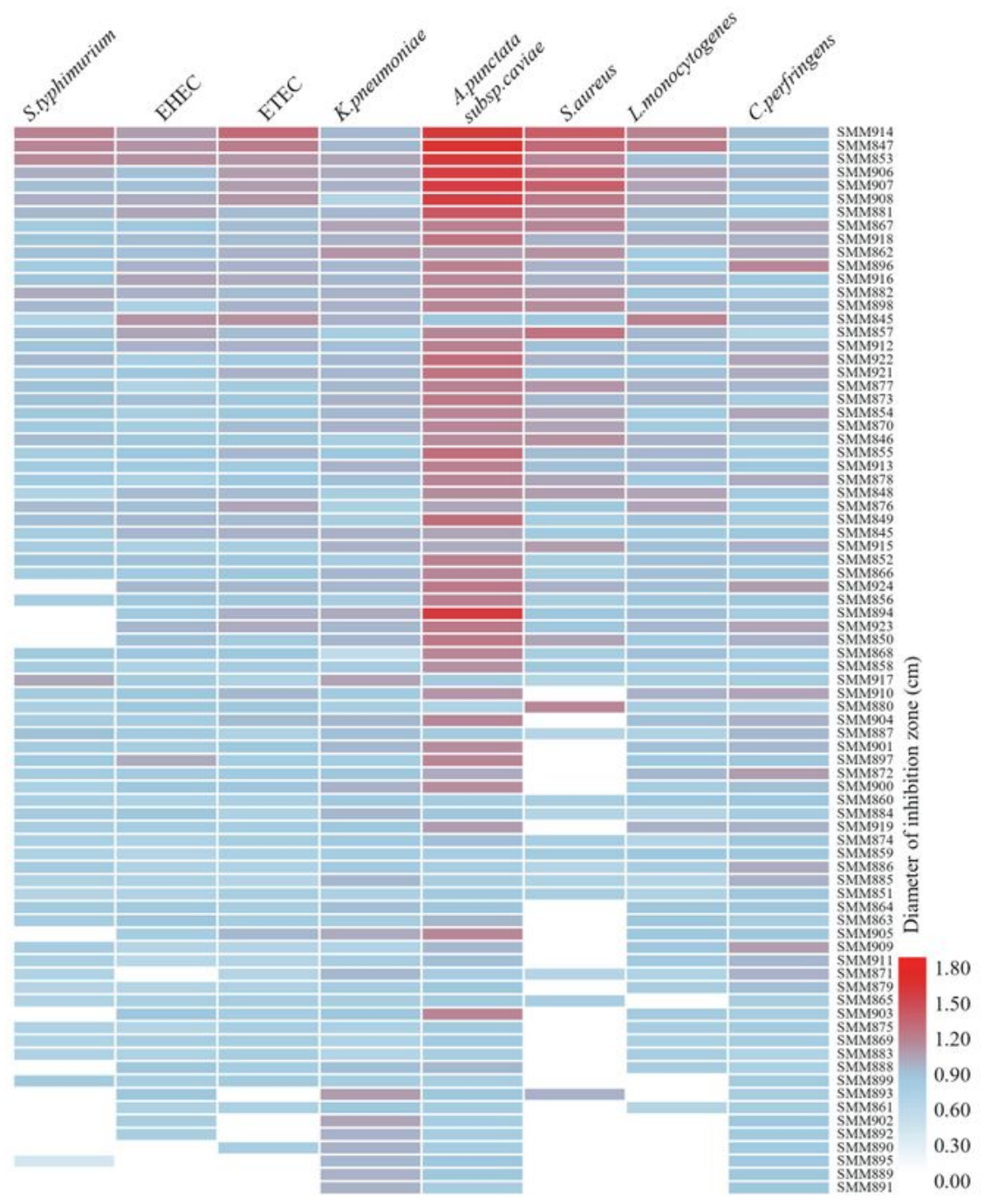

b

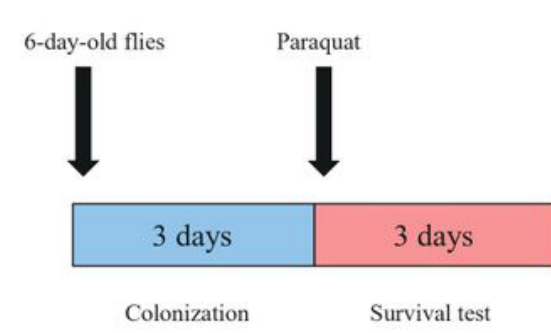

c

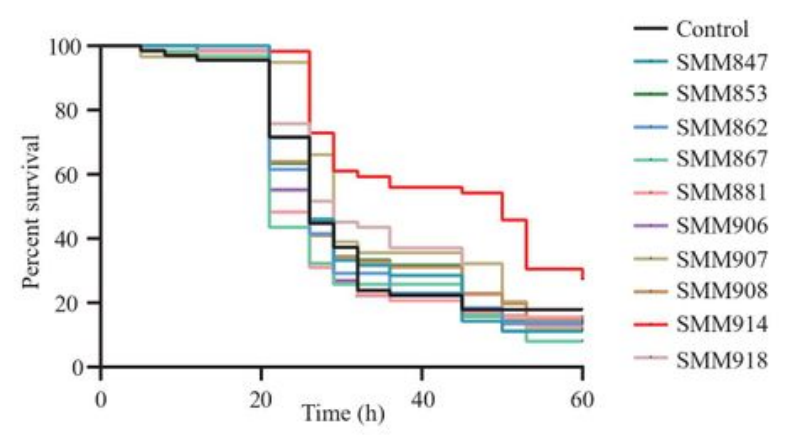

Figure 3

\section{Figure 3}

Screening of potential probiotics prior to a piglet feeding experiment. (a) Antagonistic activity against pathogens in vitro. (b) Graphical representation of experiments conducted to assess the survival rate. (c) P. pentosaceus confers protective effects upon Drosophila. Statistical differences were calculated by the log-rank test. 


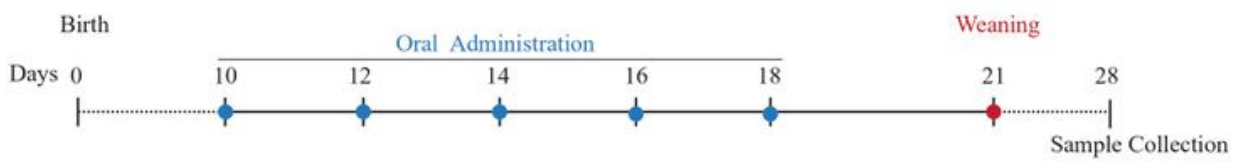

b

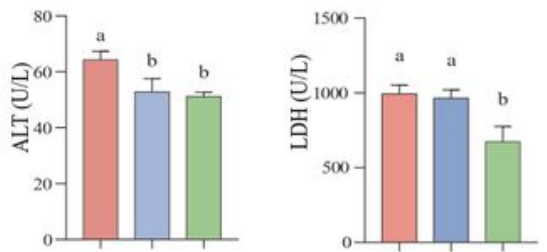

c
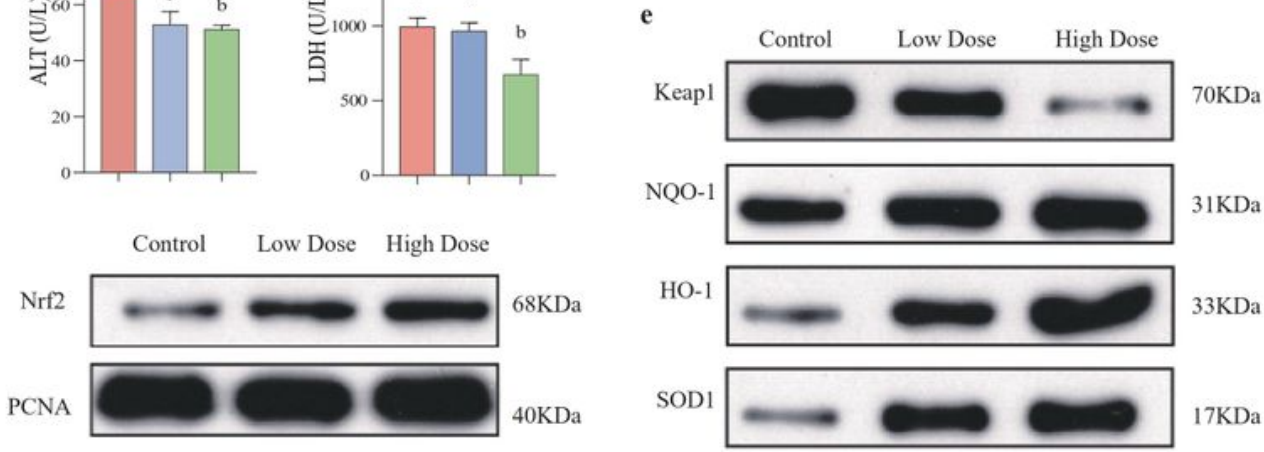

d
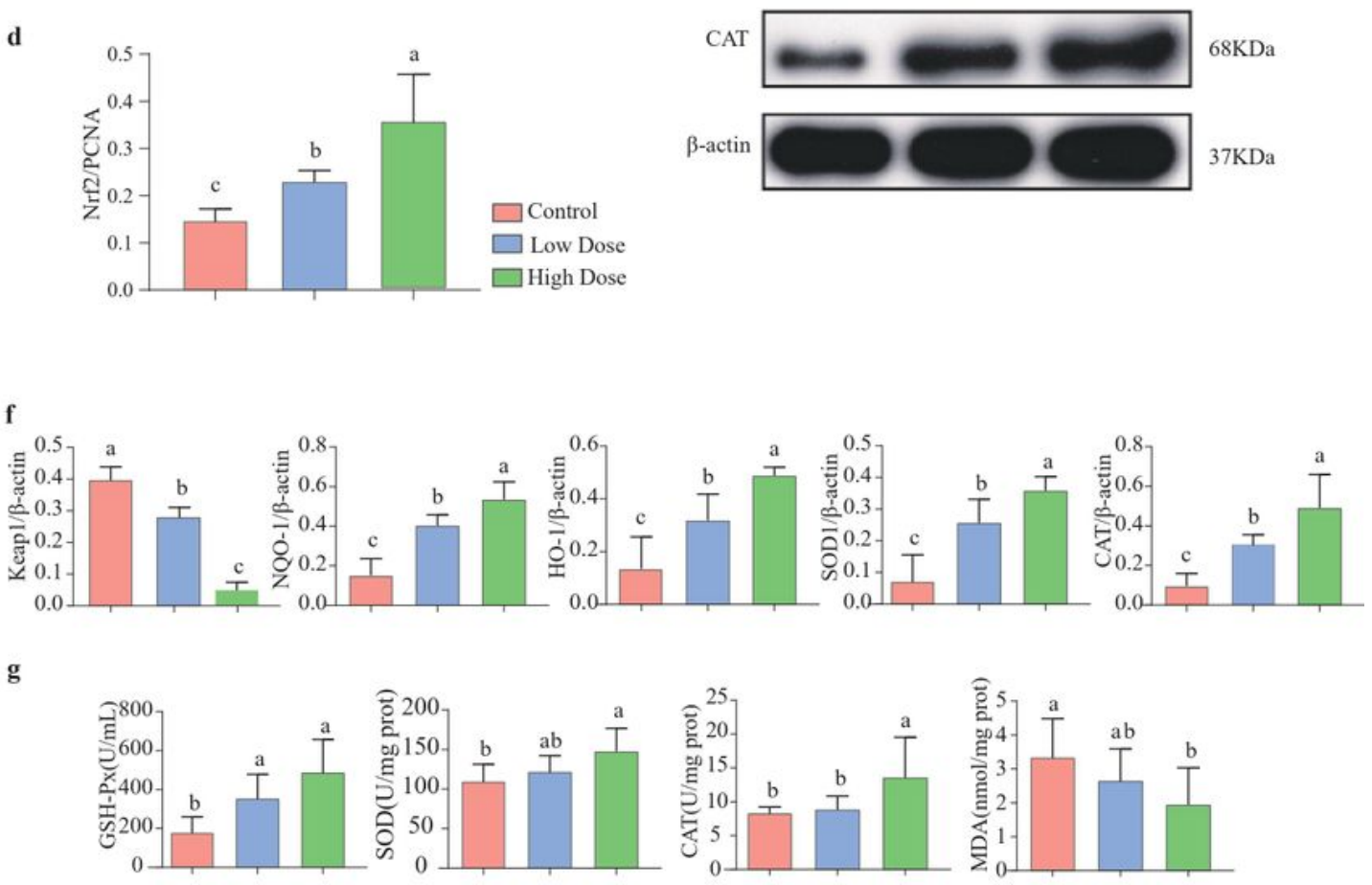

Figure 4

Figure 4

Effects of the oral administration of SMM914 on the antioxidation capacity and activation of the cytoprotective Nrf2 pathway in piglets. (a) Experimental outline in piglets $(n=18)$. Piglets were kept for 10 days after birth for adaptation. Oral administration of SMM914 was performed on days 10, 12, 14, 16 and 18 at low or high doses. At day 7 postweaning, seven piglets per treatment were randomly selected for slaughter for sample collection. (b) Alanine amiotransferase (ALT) and lactate dehydrogenase (LDH) 
were significantly decreased in piglets subjected to SMM914. (c-d) Bar graph of western blotting showing Nrf2 protein expression levels in the liver normalized to PCNA expression in the nucleus. (e-f) Western blotting analysis of antioxidant protein (Keap1, NQO-1, HO-1, SOD1 and CAT) levels in the liver and bar graph of data showing their protein expression normalized to $\beta$-actin expression in hepatic cells $(n=6)$. (g) The enzyme activities of GSH-Px, SOD, CAT and MDA were measured in liver lysates. Values of the bars stand for significant differences using oneway ANOVA followed by Duncan's multiple comparisons at $p<0.05$. Data are the mean \pm s.e. $m, n=7$.

a

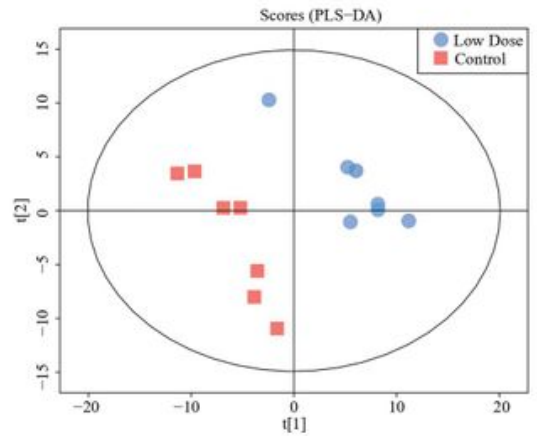

b

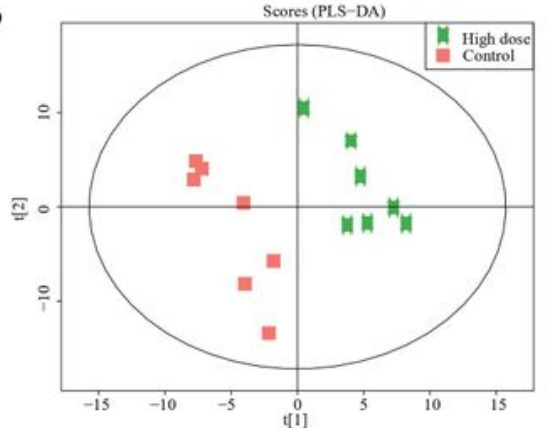

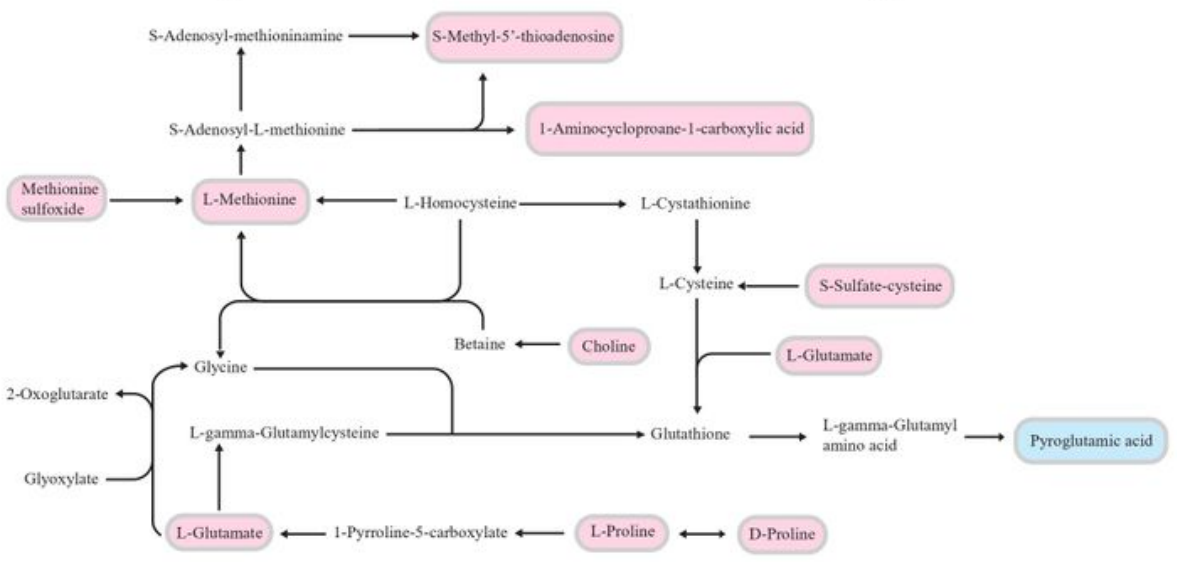

d
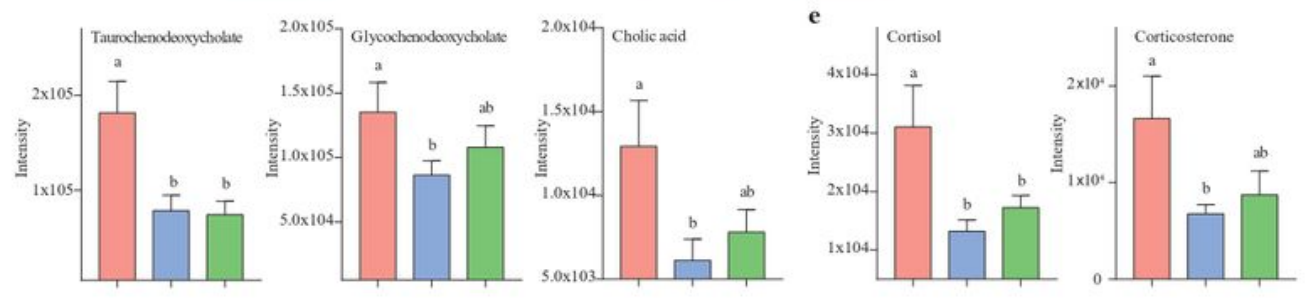

f
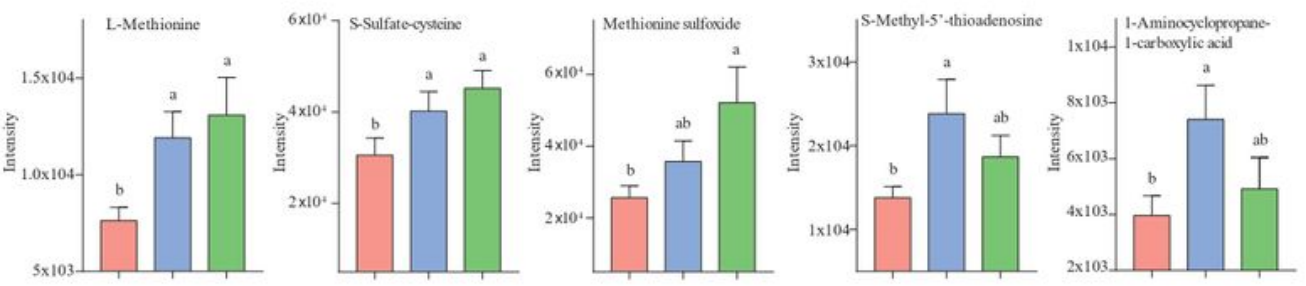

g
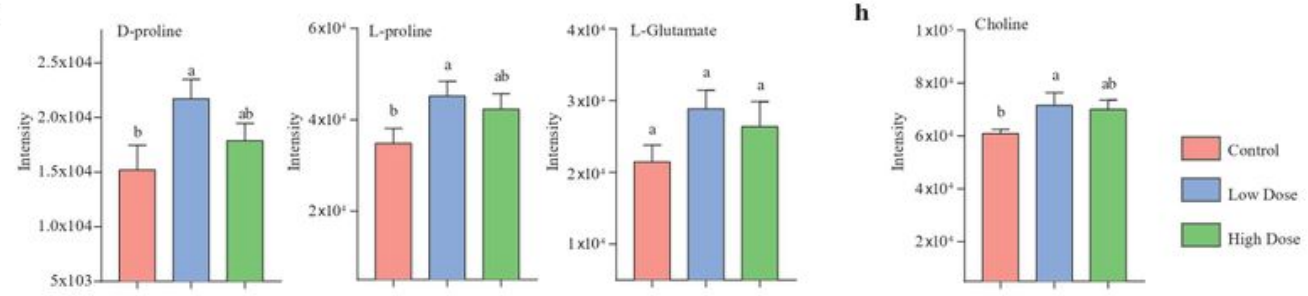

Figure 5 


\section{Figure 5}

The metabolic profile of plasma. Score plots of the PLS-DA analysis for the plasma metabolome (a) showing the scatter between the control and low-dose groups. (b) Scatter between the control and highdose groups. (c) The integrative metabolism pathway according to the KEGG pathway database. Compared with the control group, the blue metabolites represent the intensities of metabolites that were downregulated, while the red metabolites represent the intensities of metabolites that were upregulated. (d-h) The perturbed metabolism pathways and metabolites in response to SMM914 treatment mainly include glutathione metabolism; cysteine and methionine metabolism; glycine, serine and threonine metabolism; and arginine and proline metabolism. Data are the mean \pm s.e. $m, n=7$. 

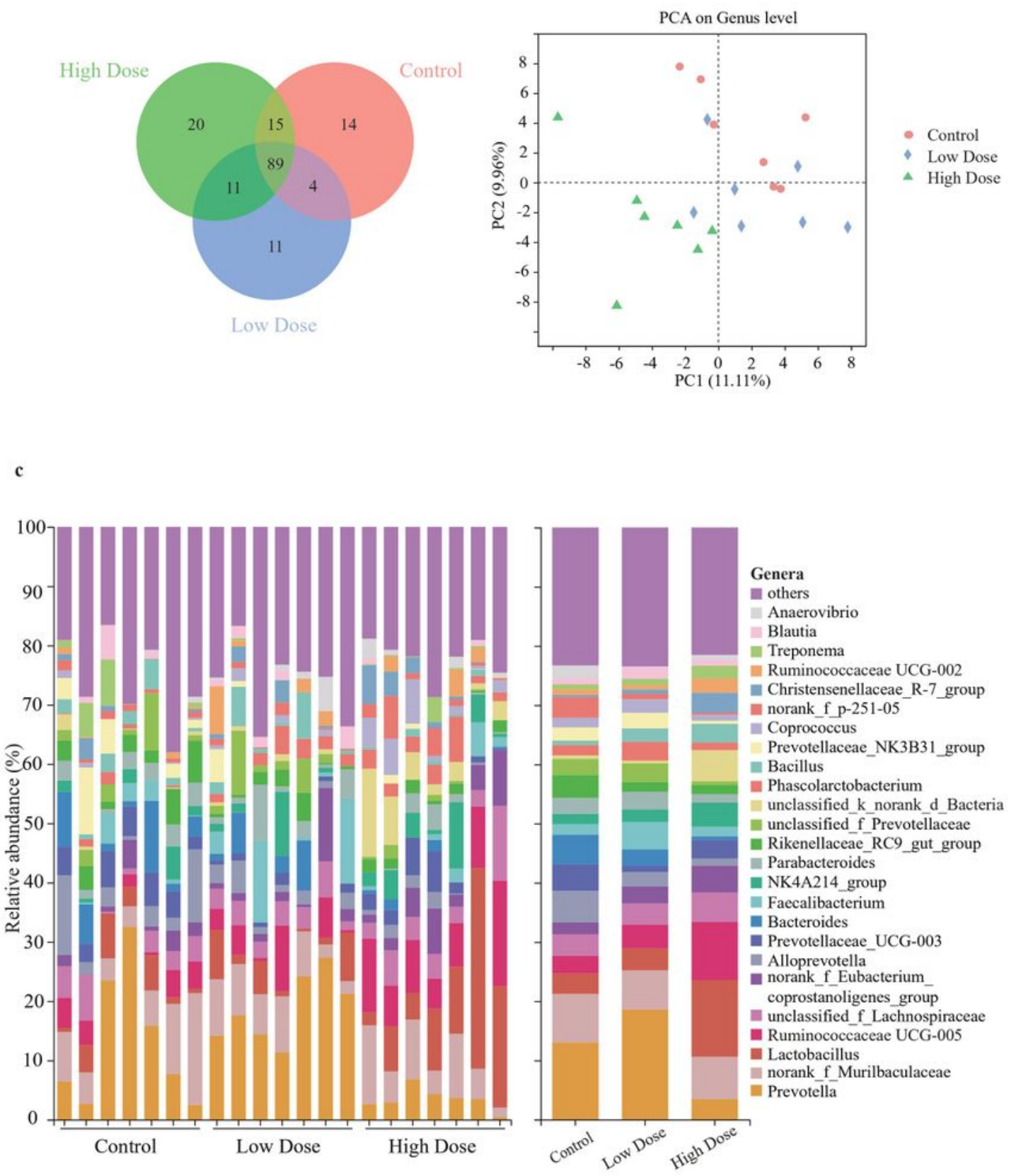

Figure 6

\section{Figure 6}

Microbial composition analysis in the colon. (a) The Venn diagram for operational taxonomic units among the control group, low-dose group and high-dose group. (b) Scatterplot of principal component analysis in bacterial communities. Principal components (PCs) 1 and 2 explain $11.11 \%$ and $9.96 \%$ of the variance, respectively (permutational multivariate analysis of variance). (c) Individual (left) and averaged (right) taxon summary of bacterial genera in colon contents. 


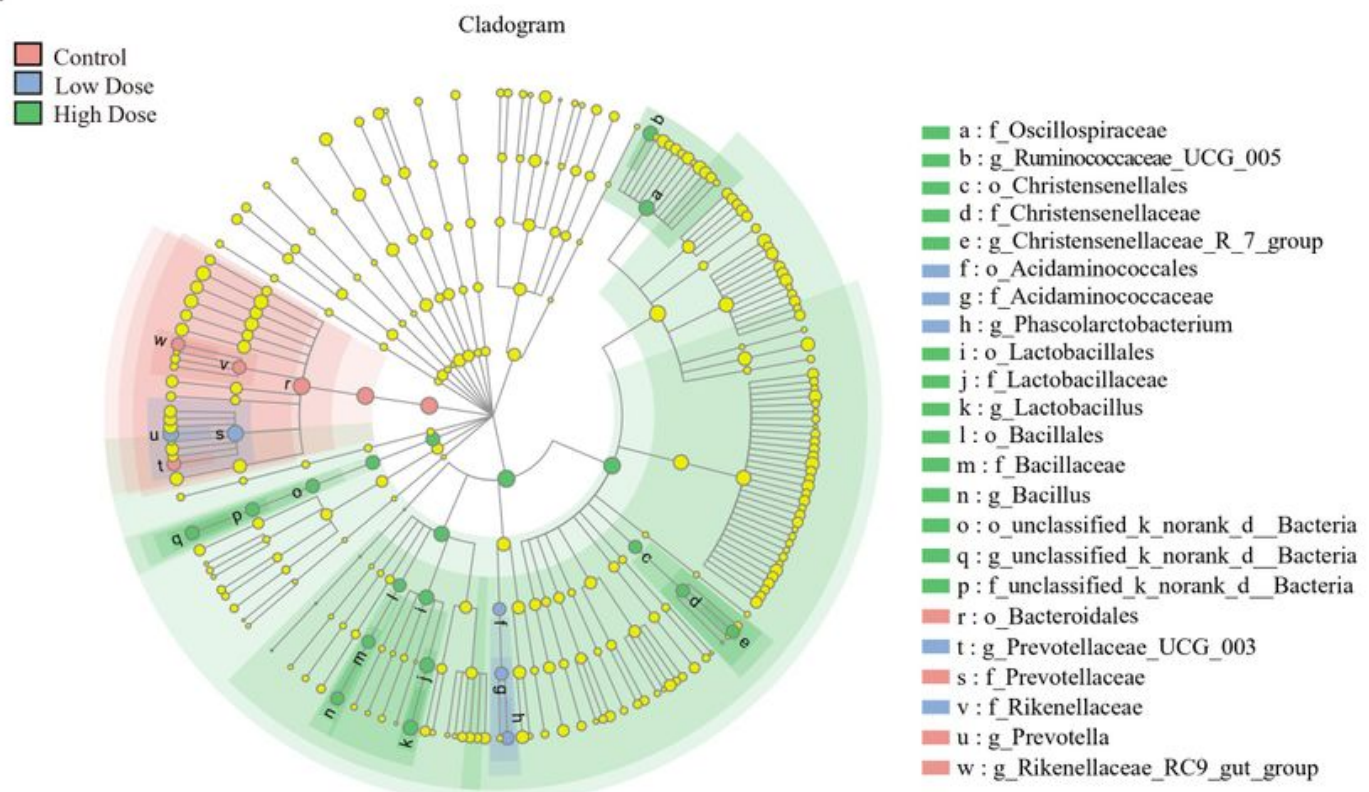

b

Wilcoxon rank-sum test bar plot on genus level

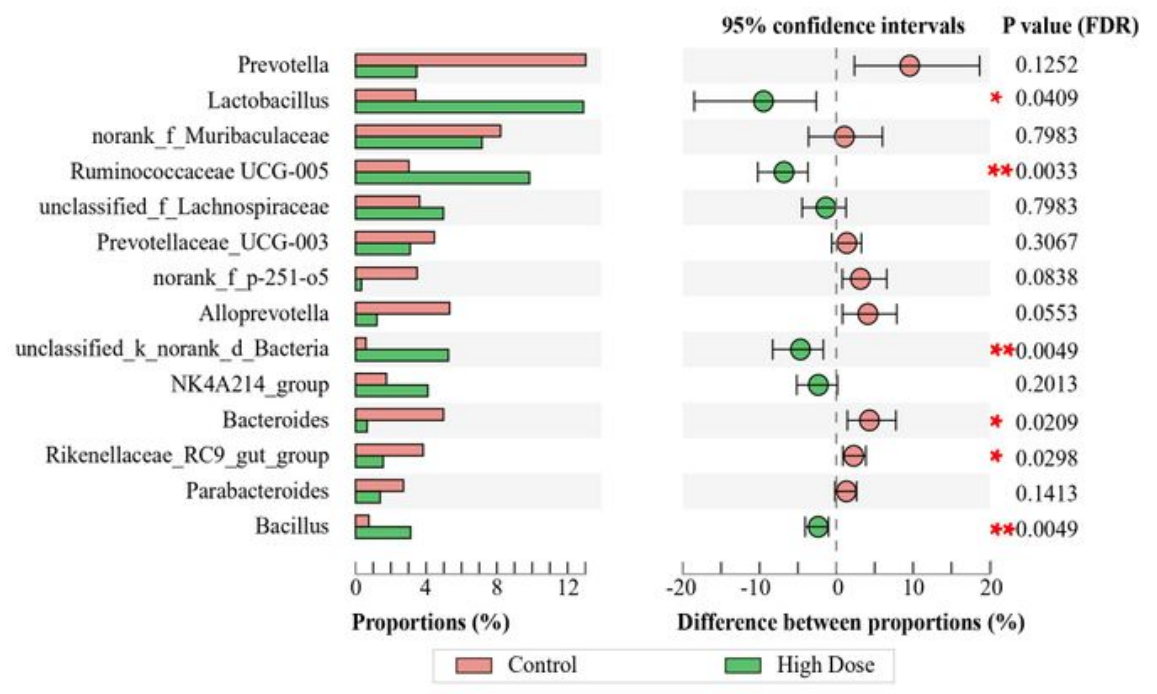

Figure 7

\section{Figure 7}

(a) Cladogram generated from Linear discriminant analysis (LDA) effect size (LEfSe) analysis for the most differentially abundant taxa with LDA scores above 4. (b) Comparison of microbial genera between high dose group and control. Significantly different domains were tested by Wilcoxon rank-sum test. *p < $0.05 ; * \star p<0.01$. 


\section{Supplementary Files}

This is a list of supplementary files associated with this preprint. Click to download.

- supplementarymaterial.zip 